\title{
A GRAPHIC FORMULATION OF NON-ISOTHERMAL CHEMICAL REACTION SYSTEMS AND THE ANALYSIS OF DETAILED BALANCED NETWORKS *
}

\author{
ZHOU FANG ${ }^{\dagger}$, ARJAN VAN DER SCHAFT $\ddagger$, AND CHUANHOU GAO $\S$
}

\begin{abstract}
In this paper, we provide a graphic formulation of non-isothermal reaction systems and show that a non-isothermal detailed balanced network system converges (locally) asymptotically to the unique equilibrium within the invariant manifold determined by the initial condition. To model thermal effects, the proposed modeling approach extends the classical chemical reaction network by adding two parameters to each direct (reaction) edge, depicting, respectively, the instantaneous internal energy change after the firing of the reaction and the variation of the reaction rate with respect to the temperature. For systems possessing thermodynamic equilibria, our modeling approach provides a compact formulation of the dynamics where reaction topology and thermodynamic information are presented simultaneously. Finally, using this formulation and the Legendre transformation, we show that non-isothermal detailed balanced network systems admit some fundamental properties: dissipativeness, the detailed balancing of each equilibrium, the existence and uniqueness of the equilibrium, and the asymptotic stability of each equilibrium. In general, the analysis and results of this work provide insights into the research of non-isothermal chemical reaction systems.
\end{abstract}

Key words. Non-isothermal chemical reaction networks, detailed balanced networks, asymptotic stability, thermodynamics, and Legendre transformation.

AMS subject classifications. 34D20, 80A30, 80A50, 93C15.

1. Introduction. Chemical reaction network $(\mathrm{CRN})$ is a simple graphic structure that has been applied extensively to modeling and analyzing complex dynamic systems, ranging from biochemistry to epidemics. The corresponding theory, called CRN theory (CRNT), has been developed in over the last decades, since the seminal papers published in 1970's by Horn and Jackson [26] and Feinberg [19]. One of the central issues in CRNT is to explore the connection between network topology and dynamical properties, including stability [1,19,26, 28,29,34,39,41], unique/multistationarity [15,20-22, 27], robustness [36, 37], and persistence ${ }^{1}[4,5,14,24]$.

Although CRNT has been very popular within biochemistry, it fails to handle chemical reactions occurring in the field of chemical engineering. The main reason is that CRNT assumes the temperature in the reaction system to be constant. However, many reactions absorb or release heat when they fire, leading to a change of temperature. A typical example is the ammonia synthesis reaction

$$
\mathrm{N}_{2}+3 \mathrm{H}_{2} \rightleftharpoons 2 \mathrm{NH}_{3}
$$

where $\mathrm{N}_{2}$ is nitrogen, $\mathrm{H}_{2}$ is hydrogen, and $\mathrm{NH}_{3}$ is ammonia. The forward reaction

* Submitted to the editors DATE.

Funding: This work is in part supported by the National Natural Science Foundation of China under Grant No. 11671418 and 12071428, and in part by the Zhejiang Provincial Natural Science Foundation of China under Grant LZ20A010002.

$\dagger$ Zhou Fang was with School of Mathematical Sciences, Zhejiang University, Hangzhou 310027, China, during this work. (zhou_fang@zju.edu.cn) Currently, he is with the Department of Biosystems Science and Engineering, ETH Zurich.

$¥$ Arjan van der Schaft is with Johann Bernoulli Institute, Faculty of Science and Engineering, University of Groningen, Netherlands. (a.j.van.der.schaft@rug.nl.)

$\S$ Chuanhou Gao is with School of Mathematical Sciences, Zhejiang University, Hangzhou 310027, China. (gaochou@zju.edu.cn)

${ }^{1}$ persistence means there is no $\omega$-limit point on the boundary of the positive quadrant. 
will release the heat of $92.4 \mathrm{KJ} / \mathrm{mol}^{2}$ while the backward reaction will absorb the same amount of heat. For this class of reactions, called here non-isothermal CRNs (the classic CRNs are called isothermal CRNs for the sake of distinction), the dynamics exhibits more complex behaviors. On the one hand, the change of temperature should be captured, often represented by the change of internal energy (see Appendix A for physical explanation of this terminology and others); on the other hand, the reaction rate coefficient is no longer a constant but a function of temperature following the Arrhenius law (or the transition state theory in a broader sense) $[8,16,31,40]$. Thus, new theory is needed to address the issues of modeling and analyzing dynamical behaviors of non-isothermal CRN systems.

A pioneering work in this regard is in Wang et al. [45, 46], investigating nonisothermal CRNs through the port-Hamiltonian modeling approach. This approach, rooted in the classical Hamiltonian equations, models the physical system by utilizing external ports and a Dirac structure. Mathematically, it amounts to define skewsymmetric tensor fields on the state space, an energy function called the Hamiltonian which is often given by the total stored energy of the system, and energy-dissipating relations. With this method, this work uses additional parameters to depict the Arrhenius law, write dynamics of isolated systems in the form of port-Hamiltonian systems, and express interconnection between target systems and environments via external ports. As a result it is shown that detailed balanced networks are dissipative with respect to availability functions of the entropy and convergent to a set of equilibria [46, Theorem 5.1 and 5.2]. However, apart from these results, many topics within the theory non-isothermal CRNs are not yet addressed. Up to now, there are still no systematic approaches to model generic non-isothermal CRN systems, especially when chemical reactions, mass fluxes, and heat exchanges are simultaneously present.

For the above reasons, the current work intends to develop basic theory for nonisothermal CRNs, including the definition of basic concepts and the derivation of basic results that are parallel to those in isothermal CRNT. To this end, we provide a systematic method to model generic non-isothermal CRN system in order to derive results going beyond those in [46]. Note that since non-isothermal CRN systems have to obey the first and second law of thermodynamics [48], thermodynamic knowledge will be key for their analysis. For the convenience of the readers, we present a review on thermodynamics in Appendix B.1 as preliminary knowledge.

Actually, it is not a new idea to utilize thermodynamics to analyze CRN systems. Within the isothermal detailed balanced CRN framework, van der Schaft et al. [41,42] established a compact formulation which exhibits at the same time the graphic structure and the aforementioned thermodynamic knowledge, by which dynamical properties can be easily characterized. A non-isothermal analog to this compact formulation is presented in [46] for isolated systems (i.e., systems with no mass flux or heat exchanges) and greatly simplifies the stability analysis in that scenario. Due to the introduction of pseudo-Helmholtz free energies, which served as Lyapunov functions for complex balanced networks [26], many studies focusing on the thermodynamic interpretations and properties of these functions have been published. Following [41,42], Rao et al. [34] applied the pseudo-Helmholtz free energy to provide a similar compact mathematical formulation of complex-balanced networks, exhibiting at the same time the graphic structure and the thermodynamic relations. From the viewpoint of statistical mechanics, Anderson and his co-workers showed that the stationary distribution of a stochastic complex-balanced (isothermal) network has a product-

\footnotetext{
${ }^{2} \mathrm{KJ}$ means kilojoule, energy unit; mol means mole, mass unit.
} 
form [7], similar to Poisson distributions, and its non-equilibrium potential leads to a pseudo-Helmholtz free energy in the classical limit case [6]. Inspired by these thermodynamic analyses, Fang and Gao [17] introduced Lyapunov function PDEs based on the stationary distribution and showed their solution to be dissipative and be able to serve as Lyapunov functions for some special cases, including, but not limited to, complex-balanced networks. The extension of classical thermodynamic concepts to non-equilibrium cases (especially to living systems) has been reviewed in [9], providing the physico-chemical basis for analyzing large-scale metabolic networks in living systems (c.f. $[9,10])$. All these studies show good examples that thermodynamic knowledge can contribute to the analysis of CRN systems. In line with these studies, we here utilize isothermal CRNT and thermodynamics to construct the basic framework of non-isothermal CRNT, and further analyze the dynamical behaviors of non-isothermal detailed balanced CRN systems. For the convenience of the reader, we also present a brief review on isothermal CRNT in Appendix B.2.

The rest of this paper is organized as follows. In section 2, we establish a graphic formulation for the modeling of non-isothermal CRN systems. Then we provide a compact dynamic formulation of non-isothermal detailed balanced networks in section 3, and further exhibit their dissipativeness. In section 4, we show the asymptotic stability of detailed balanced network systems by studying the Legendre transform of the availability function with the help of thermodynamics. Finally, section 5 concludes this paper. In the appendix, we list some mathematical notations and physical terminologies (see Appendix A), review basic knowledge about thermodynamics and isothermal CRNT (see Appendix B), and provide the detailed proofs of our main results (see Appendix C).

2. Non-isothermal CRNs. In this section, we modify the classical CRN structure so that it can cover non-isothermal reaction systems. We refer readers to Appendix B for a brief review of thermodynamics and the isothermal CRNT.

2.1. Dynamic model. We consider the same network system as stated in Appendix B. 2 but in the non-isothermal scenario. That is

$$
\alpha_{1, j} X_{1}+\cdots+\alpha_{n, j} X_{n} \stackrel{k_{j}(T)}{\longrightarrow} \tilde{\alpha}_{1, j} X_{1}+\cdots+\tilde{\alpha}_{n, j} X_{n}, \quad j=1, \ldots, r,
$$

where $X_{i}$ represents the $i$-th species in the system, $\alpha_{i, j}$ and $\tilde{\alpha}_{i, j}$ are non-negative integers called stoichiometric coefficients, the integer vectors, $\left(\alpha_{1, j}, \ldots, \alpha_{n, j}\right)^{\top}$ and $\left(\tilde{\alpha}_{1, j}, \ldots, \tilde{\alpha}_{n, j}\right)^{\top}$, are termed as complexes, and $k_{j}(T)$ are reaction rate constants at the temperature T. By the discussion in Appendix B.1, the system can be fully described by internal energy and amounts of substances, i.e., $\left(U, N_{1}, \ldots, N_{n}\right)$ or $(U, N)$, so in non-isothermal cases, we need to track the internal energy along with amounts of substances. For the $j$-th reaction, we denote the instantaneous energy change by $\Delta \mathcal{U}_{j}(U, N)$ (with the unit $J / \mathrm{mol}$ ) and assume the reaction rate coefficient, $k_{j}(T)$, to follow Eyring's formula, i.e., [16]

$$
k_{j}(T)=\tilde{k}_{j} T \exp \left\{-\frac{\Delta G_{j}^{\ddagger}(T)}{R T}\right\}=\tilde{k}_{j} T \exp \left\{-\frac{g_{j}^{\mathrm{AS}}(T)-y_{\sigma_{j}}^{\top} g(T)}{A_{v} R T}\right\},
$$

where $\tilde{k}_{j}$ is the reaction rate constant, $R$ is the Boltzmann constant, $\Delta G_{j}^{\ddagger}(T)$ (with the unit $J$ ) is the the Gibbs energy of activation, $A_{v}$ is the Avogadro constant, $g^{\mathrm{AS}}(T)$ is the free energy of the activated state, and $g(T)=\left(g_{1}(T), \ldots, g_{n}(T)\right)^{\top}$ is the free energy vector for each individual molecules (see (B.6)). Recall that the temperature $T$ 
is a function of $(U, N)$ (see Proposition B.3), and, therefore, $k_{j}(T)$ is also determined by the system state $(U, N)$. In the sequel of this paper, we will drop the arguments of $T(U, N)$ to simply the notation; please keep in mind that $T$ is a function of $(U, N)$ rather than an independent variable or a fixed constant unless specified.

In line with the above facts, we can modify the above chemical reaction scheme by the following pseudo-thermochemical equation

$\alpha_{1, j} X_{1}+\cdots+\alpha_{n, j} X_{n} \stackrel{\tilde{k}_{j}, g_{j}^{\mathrm{AS}}(T)}{\longrightarrow} \tilde{\alpha}_{1, j} X_{1}+\cdots+\tilde{\alpha}_{n, j} X_{n}, \quad \Delta \mathcal{U}_{j}(U, N), \quad j=1, \ldots, r$,

where mass variations are indicated by the stoichiometric coefficients, the instantaneous internal energy change is presented by $\Delta \mathcal{U}_{j}(U, N)$, and the transition state theory is depicted by constants $\tilde{k}_{j}$ and $g_{j}^{\mathrm{AS}}(T)$. Note that $(2.2)$ is different from the thermochemical equation, in which the variation of enthalpy under the standard condition $^{3}$ is presented instead of $\Delta \mathcal{U}_{j}(U, N)$. Clearly, (2.2) and the thermochemical equation have different physical meanings, as a consequence of which we call (2.2) the pseudo-thermochemical equation. In the sequel of this paper, we skip the arguments of the function $\Delta \mathcal{U}_{j}(U, N)$ to simplify notations; please keep in mind that $\Delta \mathcal{U}_{j}$ is a function rather than a constant term. Similar to the isothermal case, we can also view pseudo-thermochemical equations (2.2) as a graph, where complexes are vertexes, reaction arrows are edges, and $\Delta \mathcal{U}_{j}, \tilde{k}_{j}$, and $g_{j}^{\mathrm{AS}}(T)(j=1, \ldots, r)$ are parameters associated with edges. Referring to Definition B.5, we define non-isothermal CRN as follows.

Definition 2.1 (Non-isothermal CRN). A non-isothermal $C R N$ is a quintuplet $(\mathcal{S}, \mathcal{C}, \overline{\mathcal{R}}, \overline{\mathcal{K}}, \mathcal{G})$, where

- $\mathcal{S} \triangleq\left\{X_{1}, \ldots, X_{n}\right\}$ is the species set representing the considered substances,

- $\mathcal{C} \triangleq\left\{y_{1}, \ldots, y_{m}\right\}$ is the complex set consisting of all distinguished complexes where $m$ is the size of the set.

- $\overline{\mathcal{R}} \triangleq\left\{\left(y_{\sigma_{1}} \rightarrow y_{\pi_{1}}, \Delta \mathcal{U}_{1}\right), \ldots,\left(y_{\sigma_{r}} \rightarrow y_{\pi_{r}}, \Delta \mathcal{U}_{r}\right)\right\}$ is the reaction set with $\sigma_{j}$ and $\pi_{j}(1 \leq j \leq r)$ being indexes of the substrate complex and product complex of the $j$-th reaction. Particularly, for each reaction, $\sigma_{j}$ and $\pi_{j}$ should not be the same unless $y_{\sigma_{j}}$ and $y_{\pi_{j}}$ are both zero complexes.

- $\overline{\mathcal{K}} \triangleq\left(\tilde{k}_{1}, \ldots, \tilde{k}_{r}\right)$.

- $\mathcal{G} \triangleq\left(g_{1}^{\mathrm{AS}}(T), \ldots, g_{r}^{\mathrm{AS}}(T)\right)$.

Compared with the isothermal $\mathrm{CRN}(\mathcal{S}, \mathcal{C}, \mathcal{R}, \mathcal{K})$ (see Definition B.5), the nonisothermal $\mathrm{CRN}$ attaches a new term, $\Delta \mathcal{U}_{j}$, to each reaction edge, while preserving other basic structures (the species set, the complex set, and connection relations). Therefore, from the graph theory, the triplet $(\mathcal{S}, \mathcal{C}, \overline{\mathcal{R}})$ that represents the skeleton of a non-isothermal $\mathrm{CRN}$ can be alternatively described by the complex matrix, $Y \triangleq$ $\left(y_{1}, \ldots, y_{m}\right) \in \mathbb{R}^{n \times m}$, the incidence matrix $D$ of the form

$$
D_{i, j} \triangleq\left\{\begin{array}{cc}
1, & i=\pi_{j}, \\
-1, & i=\sigma_{j}, \\
0, & \text { otherwise, }
\end{array} \quad i \in\{1, \ldots, m\} \text { and } j \in\{1, \ldots, r\}\right.
$$

and the energy variation matrix $\Delta \mathcal{U} \triangleq\left(\Delta \mathcal{U}_{1}, \ldots, \Delta \mathcal{U}_{r}\right)$. Also, note that though $\mathcal{K}$ and $\overline{\mathcal{K}}$ both consist of constants, their entries have different physical meanings (see $(2.1))$; specifically, $\tilde{k}_{j}$ is a "part" of $k_{j}$.

\footnotetext{
${ }^{3}$ In chemistry, the standard condition means that the system is at one-atmosphere pressure, and the temperature is $298.15 \mathrm{~K}\left(25^{\circ} \mathrm{C}\right)$.
} 
Following mass-action kinetics, the reaction rate can be expressed as

$$
v_{j}(U, N)=\tilde{k}_{j} T \exp \left\{-\frac{g_{j}^{\mathrm{AS}}(T)-y_{\sigma_{j}}^{\top} g(T)}{A_{v} R T}\right\} \operatorname{Exp}\left(y_{\sigma_{j}}^{\top} \operatorname{Ln} N\right), \quad j=1, \ldots, r .
$$

Imitating the dynamic model in isothermal CRN, i.e., (B.18), we can write the current one to be

$$
\left(\begin{array}{c}
\dot{U} \\
\dot{N}
\end{array}\right)=\left(\begin{array}{c}
\Delta \mathcal{U} \\
\Gamma
\end{array}\right) v(U, N)
$$

where $v(U, N)=\left(v_{1}(U, N), \ldots, v_{r}(U, N)\right)^{\top}$. Note that we write the reaction rate as a function of $(U, N)$ in the above two equations, because $T$ is a function of $(U, N)$.

In this paper, we concern ourselves with analyzing the dynamic behaviors of the non-isothermal reaction system (2.4). Particularly, we will show a non-isothermal analogy to Theorem B.6. To this end, some assumptions related to the energy variation matrix $\Delta \mathcal{U}$ and the function set $\mathcal{G}$ should be made from the viewpoint of thermodynamics, which enables the network to describe open systems and paves the way for further analyses.

2.2. Thermodynamic constraints on $\Delta \mathcal{U}$ and $\mathcal{G}$. From the viewpoint of thermodynamics, for different network systems, such as isolated systems, isothermal systems, and those in-between, the matrix $\Delta \mathcal{U}$ and set $\mathcal{G}$ are different. In this subsection, we utilize thermodynamic knowledge (see Appendix B.1) to put some constraints on them according to different systems.

For isolated systems where no energy or mass flux takes place at the boundary, the internal energy is conserved based on the first law of thermodynamics. Therefore, each reaction in this case has $\Delta \mathcal{U}_{j}=0$. Yet for isothermal systems, to keep the temperature fixed, each reaction in the form of (2.2) admits

$$
\Delta \mathcal{U}_{j}=\left(y_{\pi_{j}}-y_{\sigma_{j}}\right)^{\top} u\left(T_{e}\right),
$$

where $T_{e}$ is the fixed temperature (usually the environment temperature), and $u(\cdot)$ is the vector of energies of individual molecules (see (B.4)).

Isolated systems and isothermal systems are two kinds of extreme systems, where heat exchanges are, respectively, zero and infinitely fast. For a system in-between, we can model the heat exchange process by a pseudo-thermochemical equation

$$
\emptyset \stackrel{h, A_{v} R T \ln T}{\longrightarrow} \emptyset, \quad T_{e}-T,
$$

where $\emptyset$ is the linear combination of substances with respect to a zero vector, $h$ is a constant depending on heat fluxes, and $T_{e}$ is the environmental temperature. The validity of this modeling approach is shown as follows. Such an artificial reaction takes place at the rate of $h$ and contributes to a change of the internal energy by $T_{e}-T$ after each firing (see (2.4) and (2.1)). Consequently, its effect is equivalent to the Fourier's law that describes the process of heat exchanges between two surfaces in contact. Notably, the above pseudo-thermochemical equation requires a non-isothermal CRN to involve reactions having at the same time a zero substrate complex and a zero product complex (c.f. Definition 2.1), which is not the case in isothermal CRN. Also, for this system the mass fluxes can be modeled by the following pseudo-thermochemical equations

$$
\emptyset \stackrel{\tilde{q}_{i}^{\text {in }}, A_{v} R T \ln T}{\longrightarrow} X_{i}, \quad u_{i}\left(T_{e}\right) \quad \text { and } \quad X_{i} \stackrel{\tilde{q}_{i}^{\text {out }}, A_{v} R T \ln T+g_{i}(T)}{\longrightarrow} \emptyset, \quad-u_{i}(T),
$$


where the first "reaction" brings one molecule of the $i$-th substance and $u_{i}\left(T_{e}\right)$ energy to the system at the rate of $\tilde{q}_{i}^{\text {in }}$, and the second reaction remove one molecule of the $i$-th substance and $u_{i}(T)$ energy at the rate of $\tilde{q}_{i}^{\text {out }} N_{i}$.

As far as non-isothermal CRN systems are concerned, the instantaneous internal energy change $\Delta \mathcal{U}_{j}$ of the $j$ th $(j=1, . ., r)$ real chemical reactions should be zero due to the first law of thermodynamics, which suggests the internal energy to change only in cases when mass fluxes or heat exchanges take place.

To emphasize different natures of the above-mentioned processes, we denote three reaction subsets, $\overline{\mathcal{R}}_{\mathrm{CR}}, \overline{\mathcal{R}}_{\mathrm{IO}}$, and $\overline{\mathcal{R}}_{\mathrm{HE}}$, as follows, depicting, respectively, real chemical reactions, mass fluxes, and heat exchanges.

$$
\begin{aligned}
& \overline{\mathcal{R}}_{\mathrm{CR}} \triangleq\left\{\left(y_{\sigma_{j}} \rightarrow y_{\pi_{j}}, \Delta \mathcal{U}_{j}\right) \in \overline{\mathcal{R}} \mid y_{\sigma_{j}} \neq \mathbb{O}_{n}, y_{\pi_{j}} \neq \mathbb{O}_{n}, \text { and } \Delta \mathcal{U}_{j} \text { is constant }\right\} \\
& \overline{\mathcal{R}}_{\mathrm{IO}} \triangleq\left\{\left(y_{\sigma_{j}} \rightarrow y_{\pi_{j}}, \Delta \mathcal{U}_{j}\right) \in \overline{\mathcal{R}} \mid\right.\left.\quad \text { Each element is either }\left(\mathbb{O}_{n} \rightarrow \delta_{i}, u\left(T_{e}\right)\right) \text { or }\left(\delta_{i} \rightarrow \mathbb{O}_{n},-u(T)\right), i=1, \ldots, n .\right\} \\
& \overline{\mathcal{R}}_{\mathrm{HE}} \triangleq\left\{\left(y_{\sigma_{j}} \rightarrow y_{\pi_{j}}, \Delta \mathcal{U}_{j}\right) \in \overline{\mathcal{R}} \mid y_{\sigma_{j}}=y_{\pi_{j}}=\mathbb{O}_{n}, \text { and } \Delta \mathcal{U}_{j}=T_{e}-T\right\}
\end{aligned}
$$

Denote the sizes of the above reaction subsets by $r_{\mathrm{CR}}, r_{\mathrm{IO}}$ and $r_{\mathrm{HE}}$, respectively. Therefore, the above-discussed properties of the energy variation matrix $\Delta \mathcal{U}$ and the function set $\mathcal{G}$ can be concluded by the following assumptions.

Condition 1. $\overline{\mathcal{R}}=\overline{\mathcal{R}}_{\mathrm{CR}} \bigcup \overline{\mathcal{R}}_{\mathrm{IO}} \bigcup \overline{\mathcal{R}}_{\mathrm{HE}}$. For simplicity, we assume

$$
\left(y_{\sigma_{j}} \rightarrow y_{\pi_{j}}, \Delta \mathcal{U}_{j}\right) \in \begin{cases}\overline{\mathcal{R}}_{\mathrm{CR}}, & 0<j \leq r_{\mathrm{CR}}, \\ \overline{\mathcal{R}}_{\mathrm{IO}}, & r_{\mathrm{CR}}<j \leq r_{\mathrm{CR}}+r_{\mathrm{IO}}, \\ \overline{\mathcal{R}}_{\mathrm{IO}}, & r_{\mathrm{CR}}+r_{\mathrm{IO}}<j \leq r .\end{cases}
$$

Condition 2. One of the following statements about $\Delta \mathcal{U}_{j}$ is true.

- For each reaction in $\overline{\mathcal{R}}_{\mathrm{CR}}$, we have $\Delta \mathcal{U}_{j}(U, N)=0$.

- For each reaction in $\overline{\mathcal{R}}_{\mathrm{CR}}$, we have $\Delta \mathcal{U}_{j}(U, N)=\left(y_{\pi_{j}}-y_{\sigma_{j}}\right)^{\top} u\left(T_{e}\right)$ and $r_{\mathrm{IO}}=r_{\mathrm{HE}}=0$.

Condition 3. $\Delta G_{j}^{\ddagger}=R T \ln T$ for each reaction in $\overline{\mathcal{R}}_{I O}$ and $\overline{\mathcal{R}}_{H E}$. In other words, if $y_{\sigma_{j}}=\mathbb{D}_{n}$, then $g_{j}^{\mathrm{AS}}(T)=A_{v} R T \ln T$; if $y_{\sigma_{j}}=\delta_{i}$ and $y_{\pi_{j}}=\mathbb{O}_{n}$, then $g_{j}^{\mathrm{AS}}(T)=A_{v} R T \ln T+g_{i}(T)$.

2.3. Invariant manifolds. Provided with Condition 1 , we can rewrite the incidence matrix as

$$
D=\left(D_{\mathrm{CR}}, D_{\mathrm{IO}}, D_{\mathrm{HE}}\right)
$$

and the energy variation matrix as

$$
\Delta \mathcal{U}(U, N)=\left(\Delta \mathcal{U}_{\mathrm{CR}}(U, N), \Delta \mathcal{U}_{\mathrm{IO}}(U, N), \Delta \mathcal{U}_{\mathrm{HE}}(U, N)\right),
$$

where $D_{\ell}$ and $\Delta \mathcal{U}_{\ell}$ are, respectively, the incidence matrix and the energy variation matrix with respect to the subnetwork $\overline{\mathcal{R}}_{\ell}(\ell \in\{\mathrm{CR}, \mathrm{IO}, \mathrm{HE}\})$. Therefore, we can divide the vector field of (2.4) into three parts as follows, which represent the effects of real chemical reactions, mass fluxes, and heat exchanges, respectively,

$$
\left(\begin{array}{c}
\dot{U} \\
\dot{N}
\end{array}\right)=\left(\begin{array}{c}
\Delta \mathcal{U}_{\mathrm{CR}} \\
Y D_{\mathrm{CR}}
\end{array}\right) v_{C R}(U, N)+\left(\begin{array}{c}
\Delta \mathcal{U}_{\mathrm{IO}} \\
Y D_{\mathrm{IO}}
\end{array}\right) v_{I O}(U, N)+\left(\begin{array}{c}
\Delta \mathcal{U}_{\mathrm{HE}} \\
Y D_{\mathrm{HE}}
\end{array}\right) v_{H E}(U, N) .
$$


Here, $v_{C R}(U, N)=\left(v_{1}(U, N), \ldots, v_{r_{C}}(U, N)\right)^{\top}$ depicts reaction rates of $\overline{\mathcal{R}}_{\mathrm{CR}}, v_{I O}(U, N)=$ $\left(v_{r_{C R}+1}(U, N), \ldots, v_{r_{C R}+r_{I O}}(U, N)\right)^{\top}$ depicts reaction rates of $\overline{\mathcal{R}}_{\mathrm{IO}}$, and $v_{H E}(U, N)=$ $\left(v_{r_{C R}+r_{I O}+1}(U, N), \ldots, v_{r}(U, N)\right)^{\top}$ depicts reaction rates of $\overline{\mathcal{R}}_{\mathrm{HE}}$.

Similar to the stoichiometric matrix $\Gamma=Y D$ for the isothermal case, we define the stoichiometric-like matrix as follows to indicate the potential change of the state,

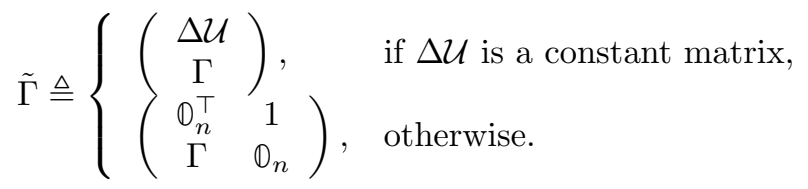

From (2.4) (also (2.5)), we can observe that the increment of the state always belongs to the linear space $\operatorname{Im} \tilde{\Gamma}$, and, therefore, can only evolve in an invariant set

$$
\left((U(0), N(0))^{\top}+\operatorname{Im} \tilde{\Gamma}\right) \bigcap \mathbb{R}_{\geq 0}^{n+1} \bigcap\left\{(U, N) \mid U \geq N^{\top} u(0)\right\}
$$

where the inequality $U \geq N^{\top} u(0)$ suggests the temperature to be non-negative. Parallel to the isothermal case, we term the linear space $\operatorname{Im} \tilde{\Gamma}$ as the stoichiometric-like subspace, the above invariant sets as the stoichiometric-like compatibility class, and the interior of the invariant set,

$$
\mathcal{P} \mathcal{S}(U(0), N(0)) \triangleq\left((U(0), N(0))^{\top}+\operatorname{Im} \tilde{\Gamma}\right) \bigcap \mathbb{R}_{>0}^{n+1} \bigcap\left\{(U, N)^{\top} \mid U>N^{\top} u(0)\right\},
$$

as the positive stoichiometric-like compatibility class.

Remark 2.2. Compared with the work in [45,46], our model $(\mathcal{S}, \mathcal{C}, \overline{\mathcal{R}}, \overline{\mathcal{K}}, \mathcal{G})$ utilizes the transition state theory to provide a much clearer interpretation to reaction kinetics and a close connection between the kinetics and thermodynamic properties (c.f. (2.3) and Condition 3), which enables the modeling approach to cover a broader class of reaction systems (see subsection 2.2) and also greatly benefit the analysis of dynamical behaviors in the sequel of this paper. In the meanwhile, our approach can also provide a clear statement of the invariant manifold of the considered dynamic system, which is also essential in the analysis of asymptotic stability.

EXAMPLE 1. We consider an isolated chemical reaction system having the following pseudo-thermochemical equations

$$
X_{1}+X_{2} \stackrel{\tilde{k}_{1}, g_{1}^{\mathrm{AS}}(T)}{\longrightarrow} X_{3}, \quad 0 ; \quad X_{3} \stackrel{\tilde{k}_{2}, g_{2}^{\mathrm{AS}}(T)}{\longrightarrow} X_{1}+X_{2}, \quad 0 .
$$

Alternatively, the pseudo-thermochemical equation can also be presented by a nonisothermal $\operatorname{CRN}(\mathcal{S}, \mathcal{C}, \overline{\mathcal{R}}, \overline{\mathcal{K}}, \mathcal{G})$ where $\mathcal{S}=\left\{X_{1}, X_{2}, X_{3}\right\}, \mathcal{C}=\left\{(1,1,0)^{\top},(0,0,1)^{\top}\right\}$,

$$
\overline{\mathcal{R}}=\left\{\left((1,1,0)^{\top} \rightarrow(0,0,1)^{\top}, 0\right),\left((0,0,1)^{\top} \rightarrow(1,1,0)^{\top}, 0\right)\right\},
$$

$\overline{\mathcal{K}}=\left(\tilde{k}_{1}, \tilde{k}_{2}\right)$ and $\mathcal{G}=\left(g_{1}^{\mathrm{AS}}(T), g_{2}^{\mathrm{AS}}(T)\right)$. Note that, in the above network, both reactions in $\overline{\mathcal{R}}$ belong to $\overline{\mathcal{R}}_{\mathrm{CR}}$, i.e., both reactions are "real" chemical reactions; also Condition 2 and Condition 3 are clearly satisfied. In this network, the incidence matrix $D$ and the complex matrix $Y$ are given by

$$
D=\left(\begin{array}{cc}
-1 & 1 \\
1 & -1
\end{array}\right), \quad Y=\left(\begin{array}{ll}
1 & 0 \\
1 & 0 \\
0 & 1
\end{array}\right)
$$


Finally by (2.4) or (2.5), we can express the dynamics as

$$
\begin{aligned}
\left(\begin{array}{c}
\dot{U} \\
\dot{N}
\end{array}\right) & =\left(\begin{array}{c}
\Delta \mathcal{U} \\
\Gamma
\end{array}\right) v(U, N) \\
& =\underbrace{\left(\begin{array}{cc}
0 & 0 \\
-1 & 1 \\
-1 & 1 \\
1 & -1
\end{array}\right)}_{\tilde{\Gamma}}\left(\begin{array}{c}
\tilde{k}_{1} T \exp \left\{-\frac{g_{j}^{\mathrm{AS}}(T)-g_{1}(T)-g_{2}(T)}{A_{v} R T}\right\} N_{1} N_{2} \\
\tilde{k}_{2} T \exp \left\{-\frac{g_{j}^{\mathrm{AS}}(T)-g_{3}(T)}{A_{v} R T}\right\} N_{3}
\end{array}\right)
\end{aligned}
$$

where the state of the system can only evolve in the stoichiometric-like compatibility class $\left((U(0), N(0))^{\top}+\operatorname{Im} \tilde{\Gamma}\right) \cap \mathbb{R}_{\geq 0}^{4} \bigcap\left\{(U, N) \mid U \geq N^{\top} u(0)\right\}$.

3. Non-isothermal detailed balanced CRNs and their dissipativeness. Recall that our goals is to extend basic results of detailed balanced system (see Theorem B.6) to the non-isothermal case. In this section, we define detailed balanced networks for non-isothermal CRNs and establish a compact dynamic formula for them by which strictly dissipativeness can be shown. The stability result and detailed balancing of each equilibrium are respectively shown in Theorem 3.5 and Corollary 3.6, which extend the first two results of Theorem B.6. The third extension regarding the asymptotic convergence to a unique positive equilibrium will be discussed in the next section.

3.1. Non-isothermal detailed balanced networks. The concept of detailed balance consists of two key components, the reversibility and the balance of reaction rates of each reversible pair at an equilibrium. We first define the reversibility of non-isothermal CRNs.

Definition 3.1 (Reversibility). We call a non-isothermal $\operatorname{CRN}(\mathcal{S}, \mathcal{C}, \overline{\mathcal{R}}, \overline{\mathcal{K}}, \mathcal{G})$ reversible, if for every reaction $\left(y_{\sigma_{j}} \rightarrow y_{\pi_{j}}, \Delta \mathcal{U}_{j}\right) \in \overline{\mathcal{R}}$ there exists a unique $\left(y_{\sigma_{\tilde{j}}} \rightarrow\right.$ $\left.y_{\pi_{\tilde{j}}}, \Delta \mathcal{U}_{\tilde{j}}\right) \in \overline{\mathcal{R}}$ such that $y_{\sigma_{j}}=y_{\pi_{\tilde{j}}}$ and $y_{\pi_{j}}=y_{\sigma_{\tilde{j}}}$. Moreover, we name $\left(y_{\sigma_{\tilde{j}}} \rightarrow\right.$ $\left.y_{\pi_{\tilde{j}}}, \Delta \mathcal{U}_{\tilde{j}}\right)$ the backward/reverse reaction of $\left(y_{\sigma_{j}} \rightarrow y_{\pi_{j}}, \Delta \mathcal{U}_{j}\right)$.

In both isothermal case and non-isothermal case, reversibility means that each reaction has a backward counterpart that switches the substrate complex and the production complex of the former reaction. (Please refer to Appendix B.2 for the reversibility of a isothermal CRN.) However, different from the case in isothermal CRNs where reversible networks must have an even number of reactions, reversible non-isothermal CRNs can have an odd number of reactions, as it can have a reaction $\left(\mathbb{O}_{n} \rightarrow \mathbb{O}_{n}, \Delta \mathcal{U}_{j}\right)$ (c.f. Definition 2.1) whose reverse reaction is itself. Provided with Condition 1, we can also observe that each pair of forward and backward reactions belong to the same reaction subset, that is if $\left(y_{\sigma_{j}} \rightarrow y_{\pi_{j}}, \Delta \mathcal{U}_{j}\right) \in \overline{\mathcal{R}}_{\ell}$ then its backward reaction also belongs to $\overline{\mathcal{R}}_{\ell}(\ell \in\{\mathrm{CR}, \mathrm{IO}, \mathrm{HE}\})$. For reactions in $\overline{\mathcal{R}}_{\mathrm{CR}}$, we further assume the activated states of forward and backward reactions in each pair to be identical.

CONDITION 4. For each pair of forward and backward chemical reactions, $\left(y_{\sigma_{j}} \rightarrow\right.$ $\left.y_{\pi_{j}}, \Delta \mathcal{U}_{j}(U, N)\right)$ and $\left(y_{\sigma_{\tilde{j}}} \rightarrow y_{\pi_{\tilde{j}}}, \Delta \mathcal{U}_{\tilde{j}}(U, N)\right)$, in $\overline{\mathcal{R}}_{\mathrm{CR}}$, there is the relation $g_{j}^{\mathrm{AS}}(T)=$ $g_{\tilde{j}}^{\mathrm{AS}}(T)$ for all $T>0$.

From the viewpoint of thermodynamics, Condition 4 means that the activated states of forward and backward reactions need to cross the same energy barrier to 
fire reactions (c.f. $[8,30])$. This condition will play an important role in establishing a compact dynamic equation that exhibits at the same time the graphic topology and thermodynamic information, which will be discussed in the next subsection.

We then define detailed balanced networks for non-isothermal CRNs by requiring reaction rates and instantaneous energy changes of reversible reactions to be balanced at some equilibrium.

DEFINITION 3.2 (Non-isothermal detailed balanced networks). A reversible nonisothermal $C R N(\mathcal{S}, \mathcal{C}, \overline{\mathcal{R}}, \overline{\mathcal{K}}, \mathcal{G})$ is detailed balanced, if at some state $\left(U^{*}, N^{*}\right)$ each pair of forward and backward reactions, $\left(y_{\sigma_{j}} \rightarrow y_{\pi_{j}}, \Delta \mathcal{U}_{j}\right)$ and $\left(y_{\sigma_{\tilde{j}}} \rightarrow y_{\pi_{\tilde{j}}}, \Delta \mathcal{U}_{\tilde{j}}\right)$, satisfy

- $v_{j}\left(U^{*}, N^{*}\right)=v_{\tilde{j}}\left(U^{*}, N^{*}\right)$, i.e., reaction rates are balanced,

- $\Delta \mathcal{U}_{j}\left(U^{*}, N^{*}\right)+\Delta \mathcal{U}_{j}\left(U^{*}, N^{*}\right)=0$, i.e., energy changes are balanced. Moreover, we name such state $\left(U^{*}, N^{*}\right)$ a detailed balanced equilibrium.

Compared with the corresponding definition for isothermal case (see Appendix B.2) non-isothermal detailed balanced networks require one more condition that the energy changes of each pair of reversible reactions are balanced. This requirement follows from the philosophy that the system is described by the internal energy and mass, and, therefore, a detailed balanced equilibrium should balance both the mass changes (by the restriction on reaction rates) and internal energy changes (by the restriction on instantaneous energy changes). The second condition of Definition 3.2 is nontrivial for open systems where $\Delta \mathcal{U}_{j}$ can vary with respect to the temperature (see the definition of $\overline{\mathcal{R}}_{\mathrm{IO}}$ ) but trivial for isolated or isothermal systems in which $\Delta \mathcal{U}_{j}$ are always constants. Moreover, to detailedly balance the pair $\left(\mathbb{D}_{n} \rightarrow \delta_{i}, u\left(T_{e}\right)\right)$ and $\left(\delta_{i} \rightarrow \mathbb{O}_{n},-u(T)\right)$ (or, $\left(\mathbb{D}_{n}, \mathbb{D}_{n}, T_{e}-T\right)$ and itself), a detailed balanced equilibrium must satisfy $T_{e}=T^{*}$, where $T^{*}$ is the system temperature at the sate $\left(U^{*}, N^{*}\right)$. In other words, a detailed balanced equilibrium satisfies

$$
T_{e}=T^{*}, \quad \text { if } r_{\mathrm{IO}}+r_{\mathrm{HE}} \neq 0 .
$$

Remark 3.3. For isolated isothermal reversible CNRs, Wegscheider's identity [47] reveals the connection between the existence of a detailed balanced equilibrium and the kinetic parameters (only including $\mathcal{K}$ ) of chemical reactions. Here, based on Condition 4, we also recur this identity for isolated non-isothermal reversible CRNs, i.e., $\overline{\mathcal{R}}=\overline{\mathcal{R}}_{\mathrm{CR}}$. Assume that the considered reversible network follows mass-action kinetics and Eyring's law (2.1), then the existence of a detailed balanced equilibrium at a given temperature $T^{*}$ is equivalent to the validity of Wegscheider's identity [25]

$$
\prod_{j=1}^{r}\left(k_{j}\left(T^{*}\right)\right)^{\lambda_{j}}=\prod_{j=1}^{r}\left(k_{\tilde{j}}\left(T^{*}\right)\right)^{\lambda_{\tilde{j}}}, \quad \forall \lambda \triangleq\left(\lambda_{1}, \ldots, \lambda_{r}\right) \in \mathbb{R}^{r}, \text { s.t. } \Gamma \lambda=\mathbb{O}_{n}
$$

where $\tilde{j}$ is the index of the reverse reaction of the $j$-th reaction. Further utilizing (2.1) and Condition 4, the above equality can be rewritten as

$$
\prod_{j=1}^{r}\left(\tilde{k}_{j}\right)^{\lambda_{j}}=\prod_{j=1}^{r}\left(\tilde{k}_{\tilde{j}}\right)^{\lambda_{\tilde{j}}}, \quad \forall \lambda \triangleq\left(\lambda_{1}, \ldots, \lambda_{r}\right) \in \mathbb{R}^{r}, \text { s.t. } \Gamma \lambda=\mathbb{D}_{n}
$$

which suggests that detailed balanced equilibria at different temperature levels are present or absent simultaneously. Particularly, if $\tilde{k}_{j}=\tilde{k}_{\tilde{j}}$ holds for any reversible reaction pair, the considered system possesses infinite many detailed balanced equilibria. 
We use the following example to exhibit the detailed balanced equilibrium in non-isothermal CRNs.

EXAMPLE 2. We still consider the non-isothermal system introduced in Example 1 whose pseudo-thermochemical equations follow (2.8). Obviously, the non-isothermat $C R N$ is reversible as the two chemical reactions switch each other's substrate complex and product complex. Provided with Condition 4, i.e., $g_{1}^{A S}(T)=g_{2}^{A S}(T)$, we can observe that (3.2) is satisfied and find a detailed balanced equilibrium $\left(U^{*}, N^{*}\right)$ with

$$
N^{*}=\left(\exp \left\{-\frac{g_{1}(1)}{A_{v} R}\right\}, \exp \left\{-\frac{g_{2}(1)}{A_{v} R}\right\}, \frac{\tilde{k}_{1}}{\tilde{k}_{2}} \exp \left\{-\frac{g_{3}(1)}{A_{v} R}\right\}\right)^{\top} \text { and } U^{*}=N^{* \top} u(1),
$$

which balances both the reaction rates and instantaneous energy changes (c.f. (2.3) and (2.2)). As a result, the network is detailed balanced.

3.2. A compact formula of detailed balanced networks. Borrowing the idea and techniques in [41,46], we provide, in this subsection, non-isothermal detailed balanced networks with compact dynamic equations that exhibit at the same time network structures and thermodynamic information. The key to providing such a formula is to rewrite reaction rates using thermodynamic quantities.

As discussed in the previous subsection, a pair of forward and backward reactions must belong to the same reaction subset provided with Condition 1. Therefore, without loss of generality, we assume the $(2 j-1)$-th reaction and $2 j$-th reaction $\left(j=1, \ldots, \frac{r_{\mathrm{CR}}+r_{\mathrm{IO}}}{2}\right)$ in a reversible network $(\mathcal{S}, \mathcal{C}, \overline{\mathcal{R}}, \overline{\mathcal{K}}, \mathcal{G})$ are a pair of forward and backward reactions. Then, we define a matrix $B \in \mathbb{R}^{m \times\left(r_{\mathrm{CR}}+r_{\mathrm{IO}}\right) / 2}$ by

$$
B_{i, j} \triangleq\left\{\begin{array}{cl}
1, & i=\pi_{2 j-1}, \\
-1, & i=\sigma_{2 j-1}, \\
0, & \text { otherwise, }
\end{array} \quad i \in\{1, \ldots, m\} \text { and } j \in\left\{1, \ldots,\left(r_{\mathrm{CR}}+r_{\mathrm{IO}}\right) / 2\right\} .\right.
$$

depicting the connection relation in a reversible network. Moreover, we term $B_{\mathrm{CR}} \in$ $\mathbb{R}^{m \times r_{\mathrm{CR}} / 2}$ as the subblock of the matrix $B$ consisting of the first $\left(r_{\mathrm{CR}} / 2\right)$ columns, and $B_{\mathrm{IO}} \in \mathbb{R}^{m \times r_{\mathrm{IO}} / 2}$ as the subblock of the matrix $B$ consisting of the last $\left(r_{\mathrm{IO}} / 2\right)$ columns. $B_{\mathrm{CR}}$ and $B_{\mathrm{IO}}$ depict respectively the connection relation in $\overline{\mathcal{R}}_{\mathrm{CR}}$ and $\overline{\mathcal{R}}_{\mathrm{IO}}$. In a reversible network, though $B_{\ell}$ is only half of the scale of the incidence matrix $D_{\ell}$ $(\ell \in\{\mathrm{CR}, \mathrm{IO}\})$, both matrices can represent the topology of $\overline{\mathcal{R}}_{\ell}$ and contain the same information.

Proposition 3.4. In a reversible non-isothermal $C R N(\mathcal{S}, \mathcal{C}, \overline{\mathcal{R}}, \overline{\mathcal{K}}, \mathcal{G})$, there hold $\operatorname{Im}(Y B)=\operatorname{Im} \Gamma$ and $\operatorname{ker}\left(B^{\top} Y^{\top}\right)=\operatorname{ker}\left(\Gamma^{\top}\right)$ if Condition 1 holds.

Proof. The result follows immediately from the fact that the matrix $B$ and the incidence matrix $D$ can be derived from each other.

For a network $(\mathcal{S}, \mathcal{C}, \overline{\mathcal{R}}, \overline{\mathcal{K}}, \mathcal{G})$ that admits a positive detailed balanced equilibrium $\left(U^{*}, N^{*}\right)$ with $U^{*}>N^{* \top} u(0)$, we term $K_{\mathrm{CR}}(T) \in \mathbb{R}^{\frac{r_{C R}}{2} \times \frac{r_{\mathrm{CR}}}{2}}$ as a diagonal matrixvalued function with

$$
\begin{aligned}
\left(K_{\mathrm{CR}}(T)\right)_{j, j} & \triangleq\left(T / T^{*}\right) \exp \left\{-\frac{g_{2 j-1}^{\mathrm{AS}}(T)}{A_{v} R T}+\frac{g_{2 j-1}^{\mathrm{AS}}\left(T^{*}\right)}{A_{v} R T^{*}}\right\} v_{2 j-1}\left(U^{*}, N^{*}\right) \\
& =\left(T / T^{*}\right) \exp \left\{-\frac{g_{2 j}^{\mathrm{AS}}(T)}{A_{v} R T}+\frac{g_{2 j}^{\mathrm{AS}}\left(T^{*}\right)}{A_{v} R T^{*}}\right\} v_{2 j}\left(U^{*}, N^{*}\right),
\end{aligned}
$$


where $T^{*}$ is the temperature at the state $\left(U^{*}, N^{*}\right)$, and the second equality follows immediately from the definition of the detailed balanced equilibrium and Condition 4. By (2.3), we can also write the diagonal element of $K_{\mathrm{CR}}(T)$ as

$$
\begin{aligned}
\left(K_{\mathrm{CR}}(T)\right)_{j, j} & =\tilde{k}_{2 j-1} T \exp \left\{-\frac{g_{2 j-1}^{A S}(T)}{A_{v} R T}\right\} \exp \left(y_{\sigma_{2 j-1}}^{\top}\left(\operatorname{Ln} N^{*}+\frac{g\left(T^{*}\right)}{A_{v} R T^{*}}\right)\right) \\
& =\tilde{k}_{2 j} T \exp \left\{-\frac{g_{2 j}^{A S}(T)}{A_{v} R T}\right\} \exp \left(y_{\pi_{2 j-1}}^{\top}\left(\operatorname{Ln} N^{*}+\frac{g\left(T^{*}\right)}{A_{v} R T^{*}}\right)\right),
\end{aligned}
$$

from which we can learn $K_{\mathrm{CR}}(T)$ is always positive definite for $T>0$. By (2.3) and the notation of $K_{\mathrm{CR}}(T)$, the rates of chemical reactions in $\overline{\mathcal{R}}_{\mathrm{CR}}$ can be expressed as

$$
\begin{aligned}
& v_{2 j-1}(U, N)=\left(K_{\mathrm{CR}}(T)\right)_{j, j} \exp \left(y_{\sigma_{2 j-1}}^{\top}\left(\operatorname{Ln} N+\frac{g(T)}{A_{v} R T}-\operatorname{Ln} N^{*}-\frac{g\left(T^{*}\right)}{A_{v} R T^{*}}\right)\right) \\
& v_{2 j}(U, N)=\left(K_{\mathrm{CR}}(T)\right)_{j, j} \exp \left(y_{\pi_{2 j-1}}^{\top}\left(\operatorname{Ln} N+\frac{g(T)}{A_{v} R T}-\operatorname{Ln} N^{*}-\frac{g\left(T^{*}\right)}{A_{v} R T^{*}}\right)\right)
\end{aligned}
$$

for $j=1, \ldots, r_{\mathrm{CR}} / 2$, and, moreover, we can conclude

$$
\begin{aligned}
Y D_{\mathrm{CR}} \cdot v_{C R}(U, N) & =\sum_{j=1}^{r_{\mathrm{CR}} / 2} Y B_{\cdot j}\left(v_{2 j-1}(U, N)-v_{2 j}(U, N)\right) \\
& =-Y B_{\mathrm{CR}} K_{\mathrm{CR}}(T) B_{\mathrm{CR}}^{\top} \operatorname{Exp}\left(Y\left(\frac{\mu}{A_{v} R T}-\frac{\mu^{*}}{A_{v} R T^{*}}\right)\right),
\end{aligned}
$$

where $\mu=\operatorname{Ln} N+\frac{g(T)}{A_{v} R T}$ is the chemical potential of the system (c.f. (B.14) and Proposition B.3) and $\mu^{*}=\operatorname{Ln} N^{*}+\frac{g\left(T^{*}\right)}{A_{v} R T^{*}}$ is the chemical potential of the system at $\left(U^{*}, N^{*}\right)$.

Similarly, we term $K_{\mathrm{IO}} \in \mathbb{R}^{\frac{r_{\mathrm{CR}}}{2}} \times \frac{r_{\mathrm{CR}}}{2}$ as a constant diagonal matrix with

$$
\left(K_{\mathrm{IO}}\right)_{j, j} \triangleq v_{2 j-1+r_{\mathrm{CR}}}\left(U^{*}, N^{*}\right)=v_{2 j+r_{\mathrm{CR}}}\left(U^{*}, N^{*}\right),
$$

where the second equality follows from the definition of the detailed balanced equilibrium. Provided with Condition 3 which suggests the kinetics of reactions in $\overline{\mathcal{R}}_{\text {IO }}$ to follow mass-action laws only, we can write diagonal elements of $K_{\mathrm{IO}}$ alternatively by

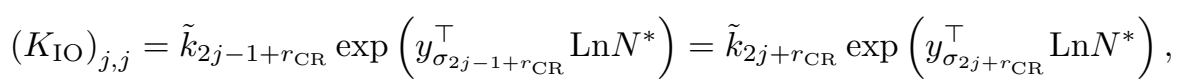

from which we can learn $K_{\mathrm{IO}}$ is also positive definite. By (2.3), Condition 3 and the notation of $K_{\mathrm{IO}}$, the rates of reactions in $\overline{\mathcal{R}}_{\mathrm{IO}}$ can be expressed as

$$
\begin{aligned}
& v_{2 j-1+r_{\mathrm{CR}}}(U, N)=\left(K_{\mathrm{IO}}(T)\right)_{j, j} \exp \left(y_{\sigma_{2 j-1+r_{\mathrm{CR}}}^{\top}}\left(\operatorname{Ln} N-\operatorname{Ln} N^{*}\right)\right), \\
& v_{2 j+r_{\mathrm{CR}}}(U, N)=\left(K_{\mathrm{IO}}(T)\right)_{j, j} \exp \left(y_{\pi_{2 j-1+r_{\mathrm{CR}}}^{\top}}^{\top}\left(\operatorname{Ln} N-\operatorname{Ln} N^{*}\right)\right)
\end{aligned}
$$

for $j=1, \ldots, r_{\mathrm{IO}} / 2$, and, therefore, we can conclude

$$
\begin{aligned}
Y D_{\mathrm{IO}} \cdot v_{I O}(U, N) & =\sum_{j=1}^{r_{\mathrm{CR}} / 2} Y B_{\cdot j+r_{\mathrm{CR}} / 2}\left(v_{2 j-1+r_{\mathrm{CR}}}(U, N)-v_{2 j+r_{\mathrm{CR}}}(U, N)\right) \\
& =-Y B_{\mathrm{IO}} K_{\mathrm{IO}} B_{\mathrm{IO}}^{\top} \operatorname{Exp}\left(Y\left(\operatorname{Ln} N-\operatorname{Ln} N^{*}\right)\right) .
\end{aligned}
$$


Also, note that the reaction in $\overline{\mathcal{R}}_{\mathrm{HE}}$, if any, points from a zero complex to itself, so $Y D_{\mathrm{HE}}$ is always zero. Moreover, provided with Condition 3, we can write the rate of the reaction in $\overline{\mathcal{R}}_{\mathrm{HE}}$ (if any) as

$$
v_{r_{\mathrm{CR}}+r_{\mathrm{IO}}+1}(U, N)=\tilde{k}_{r_{\mathrm{CR}}+r_{\mathrm{IO}}+1}\left(T_{e}-T\right)=\tilde{k}_{r_{\mathrm{CR}}+r_{\mathrm{IO}}+1}\left(T^{*}-T\right),
$$

where the second equality follows from (3.1).

Finally, by (2.5), (3.6) (3.9), and (3.10), the dynamics of a non-isothermal detailed balanced network $(\mathcal{S}, \mathcal{C}, \overline{\mathcal{R}}, \overline{\mathcal{K}}, \mathcal{G})$ can be expressed as

$$
\begin{aligned}
\dot{U}= & \Delta \mathcal{U}_{\mathrm{CR}} v_{C R}(U, N)+\Delta \mathcal{U}_{\mathrm{IO}} v_{I O}(U, N)+\tilde{k}_{r_{\mathrm{CR}}+r_{\mathrm{IO}}+1}\left(T^{*}-T\right) \chi\left(r_{\mathrm{HE}} \neq 0\right), \\
\dot{N}= & -Y B_{\mathrm{CR}} K_{\mathrm{CR}}(T) B_{\mathrm{CR}}^{\top} \operatorname{Exp}\left(Y\left(\frac{\mu}{A_{v} R T}-\frac{\mu^{*}}{A_{v} R T^{*}}\right)\right) \\
& -Y B_{\mathrm{IO}} K_{\mathrm{IO}} B_{\mathrm{IO}}^{\top} \operatorname{Exp}\left(Y\left(\operatorname{Ln} N-\operatorname{Ln} N^{*}\right)\right),
\end{aligned}
$$

if Condition 1, Condition 3, and Condition 4 hold. Compared with (2.4) and (2.5), the dynamic equation (3.11) depicts the rate and the driving force of each reaction alternatively by thermodynamic quantities, $\mu(U, N)$ and $\operatorname{Ln} N\left(=\frac{\mu(U, N)-g(T)}{A_{v} R T}\right)$. Although the formula (2.5) seems complicated in expression, it is compact with respect to information, as it exhibits at the same time the network topology, $B$, and the thermodynamic information, $\mu$. In the next subsection, some basic properties of detailed balanced networks, mainly the stability and the detailed balancing of each equilibrium, are carried out based on this formula.

In the isothermal case where $T \equiv T^{*}=T_{e}$, the dynamics (3.11) becomes

$$
\begin{aligned}
& \dot{U}=-\left(u\left(T_{e}\right)\right)^{\top} Y B K B^{\top} \operatorname{Exp}\left(Y\left(\operatorname{Ln} N-\operatorname{Ln} N^{*}\right)\right) \\
& \dot{N}=-Y B K B^{\top} \operatorname{Exp}\left(Y\left(\operatorname{Ln} N-\operatorname{Ln} N^{*}\right)\right)
\end{aligned}
$$

where

$$
K \triangleq\left(\begin{array}{cc}
K_{\mathrm{CR}}\left(T_{e}\right) & \\
& K_{I O}
\end{array}\right) .
$$

The dynamic equation (3.12) is exactly the one provided in [41] for isothermal CRNs; therefore, our dynamic equations (3.11) can be viewed as an extension of that formula (in [41]) to non-isothermal cases. Compared with the corresponding formula in [41] where $K$ is always constant (c.f. (3.12)), the dynamic equation (3.11) has a diagonal matrix-valued function $K_{\mathrm{CR}}(T)$ which can vary with respect to the temperature due to the transition state theory and exhibits the physical nature of non-isothermal systems.

In another special case where the system is isolated, i.e. $r_{\mathrm{IO}}=r_{\mathrm{HE}}=0$, the dynamics (3.11) become

$$
\begin{aligned}
& \dot{U}=0, \\
& \dot{N}=-Y B_{\mathrm{CR}} K_{\mathrm{CR}}(T) B_{\mathrm{CR}}^{\top} \operatorname{Exp}\left(Y\left(\frac{\mu}{A_{v} R T}-\frac{\mu^{*}}{A_{v} R T^{*}}\right)\right),
\end{aligned}
$$

which is exactly the formula provided in [46]. This formula can also be written in the form of a port-Hamiltonian system (see [46, (21)-(23)]). Compared with it, our formula (3.11) has additional terms, " $-Y B_{\mathrm{IO}} K_{\mathrm{IO}} B_{\mathrm{IO}}^{\top} \operatorname{Exp}\left(Y\left(\operatorname{Ln} N-\operatorname{Ln} N^{*}\right)\right)$ " and " $\tilde{k}_{r_{\mathrm{CR}}+r_{\mathrm{IO}}+1}\left(T^{*}-T\right) \chi\left(r_{\mathrm{HE}} \neq 0\right)$ ", depicting the effect of boundary fluxes and heat exchanges, and, therefore, is able to cover a broader class of systems under the same framework. 
3.3. Stability and detailed balancing of each equilibrium. We first show the stability of a detailed balanced network through Lyapunov's second method. We term

$$
\begin{aligned}
& S_{\mathcal{A}}(U, N) \\
& \triangleq-S(U, N)+\frac{\partial S\left(U^{*}, N^{*}\right)}{\partial U}\left(U-U^{*}\right)+\frac{\partial S\left(U^{*}, N^{*}\right)}{\partial N}\left(N-N^{*}\right)+S\left(U^{*}, N^{*}\right)
\end{aligned}
$$

as the availability function of the negative entropy with respect to a positive detailed balanced equilibrium $\left(U^{*}, N^{*}\right)$, which depicts the difference between the entropy and the supporting hyperplane at $\left(U^{*}, N^{*}\right)$ (c.f. $\left.[2,49]\right)$. Provided with the condition (B.3), the function $S_{\mathcal{A}}(U, N)$ is strictly positive at any positive state other than $\left(U^{*}, N^{*}\right)$ due to the strict concavity of the entropy (see Proposition B.4). The positive definiteness, together with thermodynamics underlying this function, suggests $S_{\mathcal{A}}(U, N)$ to be a good Lyapunov function candidate for investigating thermodynamic processes $[2,48$, 49]. In the isothermal case where $T \equiv T^{*}$, we can rewrite this availability function, by (B.11), as

$$
S_{\mathcal{A}}(U, N)=\frac{G\left(T^{*}, N\right)-\frac{\partial G\left(U^{*}, N^{*}\right)}{\partial N}\left(N-N^{\dagger}\right)-G\left(T^{*}, N^{*}\right)}{T^{*}}=\frac{1}{A_{v} R} G_{\mathcal{A}}(N)
$$

which implies that two functions, $S_{\mathcal{A}}(U, N)$ and $G_{\mathcal{A}}(N)$, are closely connected and that $S_{\mathcal{A}}(U, N)$ can automatically serve as a Lyapunov function for detailed balanced networks whose temperature is fixed. Moreover, $S_{\mathcal{A}}(U, N)$ is also proven to be a Lyapunov function for isolated non-isothermal detailed balanced networks [46]. In the following discussion, we further illustrate that the availability function (3.13) serves as a Lyapunov function for every non-isothermal detailed balanced network.

TheOREM 3.5. Let $(\mathcal{S}, \mathcal{C}, \overline{\mathcal{R}}, \overline{\mathcal{K}}, \mathcal{G})$ be a non-isothermal detailed balanced network admitting a positive detailed balanced equilibrium $\left(U^{*}, N^{*}\right)$, where $U^{*}=\left(N^{*}\right)^{\top} u\left(T_{e}\right)$ if $\Delta \mathcal{U}_{\mathrm{CR}}(U, N) \neq \mathbb{0}^{r} \mathrm{CR}$. Provided with (B.3), Condition 1, Condition 2, Condition 3, and Condition 4, it follows that

$$
\dot{S}_{\mathcal{A}}(U, N) \leq 0, \quad \forall(U, N) \in \begin{cases}\mathbb{R}_{>0}^{n+1} \bigcap\left\{U>N^{\top} u(0)\right\} & \text { if } \Delta \mathcal{U}_{\mathrm{CR}} \neq \mathbb{0}^{r_{\mathrm{CR}}} \\ \mathbb{R}_{>0}^{n+1} \bigcap\left\{U=N^{\top} u\left(T_{e}\right)\right\} & \text { if } \Delta \mathcal{U}_{\mathrm{CR}}=\mathbb{O}^{r} \mathrm{CR}\end{cases}
$$

where the equality holds if and only if $\nabla S_{\mathcal{A}}(U, N)=\left(\frac{1}{T^{*}}-\frac{1}{T}, \frac{\mu}{T}-\frac{\mu^{*}}{T^{*}}\right)^{\top} \in \operatorname{ker}\left(\tilde{\Gamma}^{\top}\right)$. Therefore, $S_{A}(U, N)$ is a Lyapunov function for the system (3.11) rendering the equilibrium $\left(U^{*}, N^{*}\right)$ to be stable.

Proof. The proof is shown in Appendix C.1. In short, the result follows from the fact that $B_{\mathrm{CR}} K_{\mathrm{CR}}(T) B_{\mathrm{CR}}^{\top}$ and $Y B_{\mathrm{IO}} K_{\mathrm{IO}} B_{\mathrm{IO}}^{\top}$ are both Laplacian matrices.

The above theorem shows the stability result for non-isothermal detailed balanced networks. Compare to the corresponding result for isothermal networks (the first result of Theorem B.6), both results indicate the availability function $\left(G_{\mathcal{A}}(\cdot)\right.$ or $\left.S_{\mathcal{A}}(\cdot)\right)$ to serve as Lyapunov functions, whose gradient is orthogonal to the stoichiometric(like) subspace at each non-dissipative state. Though more conditions are required in the above theorem than in Theorem B.6, these conditions are easy to verify in practical systems, and, therefore, the two stability results for respectively isothermal detailed balanced networks and non-isothermal ones are quite similar in form. Moreover, in the isothermal case where $T \equiv T_{e}=T^{*}$, the availability function $S_{\mathcal{A}}(U, N)$ is identical 
to $\frac{G_{\mathcal{A}}(N)}{A_{v} R}$ (c.f. (3.14)), and the above theorem becomes exactly the first result in Theorem B.6. Consequently, we can view Theorem 3.5 as a non-isothermal analog to the first result of Theorem B.6.

By the above theorem, we can further arrive at the detailed balancing of each positive equilibrium, which extends the second result of Theorem B.6.

Corollary 3.6. Let $(\mathcal{S}, \mathcal{C}, \overline{\mathcal{R}}, \overline{\mathcal{K}}, \mathcal{G})$ be a non-isothermal detailed balanced network with a positive detailed balanced equilibrium $\left(U^{*}, N^{*}\right)$, where $U^{*}=\left(N^{*}\right)^{\top} u\left(T_{e}\right)$ if $\Delta \mathcal{U}_{\mathrm{CR}} \neq \mathbb{O}^{r_{\mathrm{CR}}}$. If all conditions in Theorem 3.5 are satisfied, then the following statements about a positive state $\left(U^{* *}, N^{* *}\right)$, where $U^{* *}=\left(N^{* *}\right)^{\top} u\left(T_{e}\right)$ if $\Delta \mathcal{U}_{\mathrm{CR}} \neq \mathbb{O}^{r} \mathrm{CR}$, are equivalent.

1. $\left(U^{* *}, N^{* *}\right)$ is an equilibrium.

2. $\nabla S_{\mathcal{A}}\left(U^{* *}, N^{* *}\right) \in \operatorname{ker}\left(\tilde{\Gamma}^{\top}\right)$.

3. $\left(U^{* *}, N^{* *}\right)$ is a detailed balanced equilibrium.

Proof. " $1 \Rightarrow 2$ " follows immediately from Theorem 3.5 , and " $3 \Rightarrow 1$ " follows from the definition. Therefore, we only need to show " $2 \Rightarrow 3$ ".

By the second statement in the above, the definition of $\tilde{\Gamma}$, and Proposition 3.4, we can arrive at

$$
\mu^{* *} / T^{* *}-\mu^{*} / T^{*} \in \operatorname{ker}\left(B^{\top} Y^{\top}\right) .
$$

where $T^{* *}$ and $\mu^{* *}$ are the temperature and chemical potentials at $\left(U^{* *}, N^{* *}\right)$.

Balance of reaction rates: For each pair of forward and backward reactions in $\overline{\mathcal{R}}_{\mathrm{CR}}$, the relation (3.15) suggest $\left(y_{\sigma_{2 j-1}}-y_{\pi_{2 j-1}}\right)^{\top}\left(\frac{\mu^{* *}}{T^{* *}}-\frac{\mu^{*}}{T^{*}}\right)=0$ for $j=$ $1, \ldots, r_{\mathrm{CR}} / 2$, and, therefore,

$$
\begin{array}{rlr}
v_{2 j-1}\left(U^{* *}, N^{* *}\right) & =\left(K_{\mathrm{CR}}(T)\right)_{j, j} \exp \left(y_{\sigma_{2 j-1}}^{\top}\left(\frac{\mu^{* *}}{T^{* *}}-\frac{\mu^{*}}{T^{*}}\right)\right) & \\
& =\left(K_{\mathrm{CR}}(T)\right)_{j, j} \exp \left(y_{\pi_{2 j-1}}^{\top}\left(\frac{\mu^{* *}}{T^{* *}}-\frac{\mu^{*}}{T^{*}}\right)\right) \quad j=1, \ldots, r_{\mathrm{CR}} / 2 \\
& =v_{2 j}\left(U^{* *}, N^{* *}\right), \quad j=
\end{array}
$$

where the first and last equality follows from (3.4) and (3.5) respectively. Thus, we show balance of reaction rates for each pair of forward and backward reactions in $\overline{\mathcal{R}}_{\mathrm{CR}}$. For each pair of reversible reactions in $\overline{\mathcal{R}}_{\mathrm{IO}}$ (if any), we can similarly prove the result by (3.15), (3.1), (3.7), and (3.8). For the reaction in $\overline{\mathcal{R}}_{\mathrm{HE}}$, the result is trivial, as its backward reaction is itself.

Balance of energy changes: For each pair of forward and backward reactions in $\overline{\mathcal{R}}_{\mathrm{CR}}$, balance of energy changes is trivial, as $\Delta \mathcal{U}_{j}\left(j=1, \ldots, r_{\mathrm{CR}}\right)$ are constants. For each pair in $\overline{\mathcal{R}}_{\mathrm{IO}}$ and $\overline{\mathcal{R}}_{\mathrm{HE}}$ (if any), balance of energy changes follows immediately from $T^{* *}=T^{*}=T_{e}$ (c.f. (3.1)) and the restrictions of $\Delta \mathcal{U}_{j}$ in $\overline{\mathcal{R}}_{\mathrm{IO}}$ and $\overline{\mathcal{R}}_{\mathrm{HE}}$.

Given the analysis above, we show " $2 \Rightarrow 3$ " which proves the result.

4. Asymptotic stability of detailed balanced network. In this section, we show the asymptotic stability of a detailed balanced network to the unique equilibrium in each invariant manifold, which extend the third result of Theorem B.6. From Theorem 3.5 and Corollary 3.6, we can learn that the Lyapunov function $S_{\mathcal{A}}(U, N)$ dissipates at any positive state except the detailed balanced equilibrium. Therefore, by the second Lyapunov's method, showing the asymptotic stability of the system is equivalent to proving the existence and uniqueness of the detailed balanced equilibrium in each positive stoichiometric-like compatibility class. 
4.1. Legendre transformation and the proof scheme. Geometrically, the fact that the gradient of the convex function $S_{\mathcal{A}}(U, N)$ is orthogonal to the stoichiometriclike subspace at a positive detailed balanced equilibrium (see Corollary 3.6) suggests the equilibrium to be a minimum point of the Lyapunov function in the corresponding positive stoichiometric-like compatibility class. Therefore, a straightforward idea to investigate asymptotic stability is to check whether the convex function $S_{\mathcal{A}}(U, N)$ has a unique minimum point in an invariant set $\mathcal{P} \mathcal{S}\left(U^{o}, N^{o}\right)$. However, this idea is not easy to realize, because $S_{\mathcal{A}}(U, N)$ takes finite values at finite boundary points (c.f. (B.10)), and we cannot tell if $S_{\mathcal{A}}(U, N)$ has a minimum value point in such a case.

Instead, we study this problem by investigating the Legendre transformation of the Lyapunov function $S_{\mathcal{A}}(U, N)$. The Legendre transformation switches the positions of a function's independent variables and derivatives, as a consequence of which it can preserve geometric relations between these variables while possibly generate a function that is unbounded at any boundary. In what follows, we flesh out the details of the scheme of our analysis.

First, we denote the Legendre transformation of $S_{\mathcal{A}}(U, N)$ by

$$
\mathcal{L}(\beta, \gamma) \triangleq-S_{\mathcal{A}}(U, N)+\beta U+\gamma^{\top} N
$$

where $\beta=\frac{\partial S_{\mathcal{A}}(U, N)}{\partial U}=\frac{1}{T^{*}}-\frac{1}{T}, \gamma=\left(\frac{\partial S_{\mathcal{A}}(U, N)}{\partial N}\right)^{\top}=\frac{\mu}{T}-\frac{\mu^{*}}{T^{*}}$, and the definition domain is $\mathfrak{D} \triangleq\left(-\infty, \frac{1}{T^{*}}\right) \otimes \mathbb{R}^{n}$. By denoting $T^{o}$ and $\mu^{o}$ the temperature and chemical potentials at $\left(U^{o}, N^{o}\right)$, we further modify the Legendre function by shifting it at $\left(\beta^{o}, \gamma^{o}\right) \triangleq\left(\frac{1}{T^{*}}-\frac{1}{T^{o}}, \frac{\mu^{o}}{T^{o}}-\frac{\mu^{*}}{T^{*}}\right)$ and obtain

$$
\mathcal{L}_{\mathcal{A}}(\beta, \gamma)=\mathcal{L}(\beta, \gamma)-\left(\beta-\beta^{o}\right) U^{o}-\left(\gamma-\gamma^{o}\right)^{\top} N^{o}-\mathcal{L}\left(\beta^{o}, \gamma^{o}\right)
$$

Here, we term the modified function as $\mathcal{L}_{\mathcal{A}}(\cdot)$, because it is also an availability function of the Legendre transformation with respect to $\left(\beta^{o}, \gamma^{o}\right)$. Note that functions' convexities are preserved under both the Legendre transformation and the availability function transformation. Therefore, $L(\beta, \gamma)$ and $L_{\mathcal{A}}(\beta, \gamma)$ are both strictly convex. In an invariant set $\mathcal{P S}\left(U^{o}, N^{o}\right)$ where $U^{o}=\left(N^{o}\right)^{\top} u\left(T_{e}\right)$ if $\Delta \mathcal{U}_{\mathrm{CR}} \neq \mathbb{0}^{r} \mathrm{CR}$, Corollary 3.6 suggests a positive state $\left(U^{* *}, N^{* *}\right)$ to be detailed balanced if and only if

$$
\left(\begin{array}{l}
\beta^{* *} \\
\gamma^{* *}
\end{array}\right) \triangleq\left(\begin{array}{l}
\frac{1}{T^{* *}}-\frac{1}{T^{* *}} \\
\frac{\mu^{* *}}{T^{* *}}-\frac{\mu^{*}}{T^{*}}
\end{array}\right) \in \mathfrak{D} \cap \operatorname{ker}\left(\tilde{\Gamma}^{\top}\right)=\mathfrak{D} \cap(\operatorname{Im} \tilde{\Gamma})^{\perp}
$$

and

$$
\nabla \mathcal{L}_{\mathcal{A}}\left(\beta^{* *}, \gamma^{* *}\right)=\left(\begin{array}{c}
U^{* *}-U^{o} \\
N^{* *}-N^{o}
\end{array}\right) \in \operatorname{Im} \tilde{\Gamma}
$$

where $T^{* *}$ and $\mu^{* *}$ are respectively the temperature and chemical potentials at the state $\left(U^{* *}, N^{* *}\right)$. From the above relations, we can conclude by the convexity of $\mathcal{L}_{\mathcal{A}}(\cdot)$ that $\left(\beta^{* *}, \gamma^{* *}\right)$ is a minimum point of $\mathcal{L}_{\mathcal{A}}(\beta, \gamma)$ in the region $\mathfrak{D} \cap(\operatorname{Im} \tilde{\Gamma})^{\perp}$ if and only if $\left(U^{* *}, N^{* *}\right)$ is a detailed balanced equilibrium. Thus, showing the existence and uniqueness of a detailed balanced equilibrium is equivalent to proving the existence and uniqueness of a minimum point of $\mathcal{L}_{\mathcal{A}}(\beta, \gamma)$ in the region $\mathfrak{D} \cap(\operatorname{Im} \tilde{\Gamma})^{\perp}$. A straightforward way to verify it is to show $\mathcal{L}_{\mathcal{A}}(\beta, \gamma)$ to go to infinity at boundary points. Thanks to thermodynamic knowledge, especially the low temperature and high temperature limit of thermodynamic quantities, such analysis is feasible (see Proposition 4.1). 
In the isothermal case, the existence and uniqueness of a detailed balanced equilibrium in a positive stoichiometric compatibility class $\left(N^{o}+\operatorname{Im} \Gamma\right) \cap \mathbb{R}_{>0}^{n}$ is shown by investigating an auxiliary function (c.f. [22])

$$
\psi(\gamma)=\left(N^{*}\right)^{\top} \operatorname{Exp}(\gamma)-\gamma^{\top} N^{o} .
$$

Note that the Legendre transformation of the pseudo-Helmholtz free energy is

$$
\mathcal{L}_{G_{\mathcal{A}}}(\gamma) \triangleq-G_{\mathcal{A}}(N)+\gamma^{\top} N=\left(N^{*}\right)^{\top} \operatorname{Exp}(\gamma)-N^{*},
$$

where $\gamma=\nabla G_{\mathcal{A}}(N)$, and its shifted function at $\gamma=\operatorname{Ln} N^{o}-\operatorname{Ln} N^{*}$ is given by

$$
\begin{aligned}
\mathcal{L}_{\mathcal{A}, G_{\mathcal{A}}}(\gamma) & \triangleq \mathcal{L}_{G_{\mathcal{A}}}(\gamma)-\gamma^{\top} \nabla \mathcal{L}_{G_{\mathcal{A}}}\left(\gamma^{o}\right)-\mathcal{L}_{G_{\mathcal{A}}}\left(\gamma^{o}\right) \\
& =\left(N^{*}\right)^{\top} \operatorname{Exp}(\gamma)-\gamma^{\top} N^{o}+\left(N^{*}\right)^{\top} \operatorname{Exp}\left(\gamma^{o}\right),
\end{aligned}
$$

which only differs from the auxiliary function $\psi(\gamma)$ in a constant $\left(N^{*}\right)^{\top} \operatorname{Exp}\left(\gamma^{o}\right)$. Therefore, though not pointed out explicitly in [22], the Legendre transformation also governs the asymptotic stability analysis of isothermal detailed balanced networks.

4.2. Asymptotic stability. Following the proof scheme introduced in the previous subsection, we can arrive at the main results in this paper.

First, we point out that the variables $T$ and $N$ can be expressed as

$$
\begin{aligned}
T & =\left(\frac{1}{T^{*}}-\beta\right)^{-1}, \\
N_{i} & =\exp \left(\frac{\gamma+\frac{\mu^{*}}{T^{*}}-\frac{g_{i}(T)}{T}}{A_{v} R}\right)=Z_{i}(T) \exp \left(\frac{\gamma+\frac{\mu^{*}}{T^{*}}}{A_{v} R}\right), \quad i=1, \ldots, n .
\end{aligned}
$$

Given the above expressions, we further use Condition 5 to show the unboundedness of $\mathcal{L}_{A}(\beta, \gamma)$ at any boundary.

Proposition 4.1. Provided with (B.3), Condition 5, and $U^{o}>\left(N^{o}\right)^{\top} u(0)$, the function $\mathcal{L}_{A}(\beta, \gamma)$ satisfy

$$
\begin{array}{rlrl}
\lim _{(\beta, \gamma) \rightarrow\left(\beta^{b-}, \gamma^{b}\right)} \mathcal{L}_{\mathcal{A}}(\beta, \gamma) & =+\infty, & \forall\left(\beta^{b}, \gamma^{b}\right) \in\left\{\frac{1}{T^{*}}\right\} \otimes \mathbb{R}^{n}, \\
\lim _{\theta \rightarrow+\infty} \mathcal{L}_{\mathcal{A}}(\theta \bar{\beta}, \theta \bar{\gamma})=+\infty, & \forall(\bar{\beta}, \bar{\gamma}) \in\left((-\infty, 0) \otimes \mathbb{R}^{n}\right) /\left(0, \mathbb{D}_{n}\right) .
\end{array}
$$

Proof. The proof may be found in Appendix C.2.

Then we prove the existence and uniqueness of a minimum point in $\mathfrak{D} \cap \operatorname{ker}\left(\tilde{\Gamma}^{\top}\right)$. set

Proposition 4.2. Provided with (B.3), Condition 5, and $U^{o}>\left(N^{o}\right)^{\top} u(0)$, the

$$
\mathfrak{S}_{c} \triangleq\left\{(\beta, \gamma) \in \mathfrak{D} \cap \operatorname{ker}\left(\tilde{\Gamma}^{\top}\right) \mid \mathcal{L}_{A}(\beta, \gamma) \leq \mathcal{L}_{A}\left(0, \mathbb{O}_{n}\right)\right\}
$$

is non-empty, convex, and closed.

Proof. See details in Appendix C.2.

Proposition 4.3. Provided with (B.3), Condition 5 (in Appendix B.1), and $U^{o}>\left(N^{o}\right)^{\top} u(0)$, the set $\mathfrak{S}_{c}$ is compact.

Proof. See details in Appendix C.2. 
Proposition 4.4. Provided with (B.3), Condition 5, and $U^{o}>\left(N^{o}\right)^{\top} u(0)$, the shifted Legendre function $\mathcal{L}_{A}(\beta, \gamma)$ has a unique minimum in $\mathfrak{D} \cap \operatorname{ker}\left(\tilde{\Gamma}^{\top}\right)$.

Proof. See details in Appendix C.2.

Finally, we show the existence and uniqueness of a detailed balanced equilibrium in each positive stoichiometric compatibility class and the asymptotic stability of the equilibrium.

TheOREM 4.5. Let $(\mathcal{S}, \mathcal{C}, \overline{\mathcal{R}}, \overline{\mathcal{K}}, \mathcal{G})$ be a non-isothermal detailed balanced network with a positive detailed balanced equilibrium $\left(U^{*}, N^{*}\right)$, where $U^{*}=\left(N^{*}\right)^{\top} u\left(T_{e}\right)$ if $\Delta \mathcal{U}_{\mathrm{CR}} \neq \mathbb{O}^{r} \mathrm{CR}$. Provided with (B.3) and Conditions 1-5, there exists a unique positive detailed balanced equilibrium in each positive stoichiometric-like compatibility class $\mathcal{P S}\left(U^{o}, N^{o}\right)$, where

- $U^{o}>\left(N^{o}\right)^{\top} u(0)$,

- $U^{o}=\left(N^{o}\right)^{\top} u\left(T_{e}\right)$ if $\Delta \mathcal{U}_{\mathrm{CR}} \neq \mathbb{0}^{r} \mathrm{CR}$.

Moreover, this equilibrium is locally asymptotically stable.

Proof. The detailed proof is shown in Appendix C.2.

4.3. Some future remarks. Theorem 4.5 extends the third result in Theorem B.6, and, thus, non-isothermal detailed balanced networks inherit all results in Theorem B.6: the dissipativeness (Theorem 3.5), the detailed balancing of each equilibrium (Corollary 3.6), the existence and uniqueness of the equilibrium (Theorem 4.5), and the asymptotic stability (Theorem 4.5).

From the previous discussions, we can observe Condition 5 (in Appendix B.1), which suggests the unboundedness of each partition function $Z_{i}(\cdot)$, plays an important role in showing Theorem 4.5 by guaranteeing the unboundedness of the modified Legendre transformation $\mathcal{L}_{A}(\beta, \gamma)$ at any boundary. Note that this condition is absent in any result prior to this section; therefore, in this paper, it serves specifically for the asymptotic stability problem. Moreover, one can also observe that all propositions concerning $\mathcal{L}_{A}(\beta, \gamma)$ in Appendix C.2 are independent of the specific structure of a non-isothermal CRN and purely thermodynamic knowledge, which implies that thermodynamics can help researchers gain insights into CRNT.

Also, we need to emphasize that the asymptotic stability shown in Theorem 4.5 is a local result rather than a global one. It is caused by the failure of the second Lyapunov method to preclude boundary $\omega$-limit points provided that the Lyapunov function is finite at finite boundaries and can be non-dissipative on them. It still remains an open problem whether a non-isothermal detailed balanced network is globally asymptotically stable or not. To further investigate this problem, researchers are required to study the persistence of non-isothermal detailed balanced networks, i.e., to check whether or not boundary $\omega$-limit points exist. Some methodologies $[4,5,14,24]$ that succeed in investigating the persistence of isothermal CRNs can be helpful to this problem.

Notwithstanding the above limitations, our current modeling method still has enough advantages compared to the existing one, the port-Hamiltonian approach $[45,46]$. The latter models non-isothermal detailed balanced chemical reaction systems in the form of port-Hamiltonian systems (see [46, (38)]) and captures stability through the passivity structure of the Hamiltonian systems, but fails to discuss the asymptotic stability of a detailed balanced equilibrium. However, the current approach yields deeper results in understanding the dynamics of non-isothermal reaction network systems and about the stable behavior of detailed balanced equilibria. As stated in Remark 2.2, it utilizes the transition state theory to characterize the kinetics 
that could provide a much clearer interpretation to reaction kinetics and a close connection between the kinetics and thermodynamic properties. Based on these, a clear statement about the invariant manifold and the geometry of equilibrium sets may be obtained, which together with thermodynamic knowledge, particularly with the high temperature and low temperature limit, can serve for reaching the asymptotic stability of each detailed balanced equilibrium. In addition, the current method could model three parts of an open reaction network system, i.e., non-isothermal chemical reaction networks, boundary inflow and outflow, and heat exchange, in the same framework, which also greatly benefit the analysis of dynamical behaviors. However, the work of Wang et. al. [46] cannot model these three parts simultaneously using the same approach. This fact also suggests that our current modeling approach is suitable for describing a broader class of reaction systems. Naturally, it needs to be pointed out that in the special case of isolated reaction network systems the mentioned two modeling methods yield the same dynamic equations.

5. Conclusion. In this paper, we provide a graphic formulation for modeling non-isothermal chemical reaction systems based on the classical CRNT and apply it to analyzing dynamic properties of detailed balanced network systems. To model thermal effects, we first extend the isothermal CRN by adding two parameters to each (reaction) edge depicting respectively the instantaneous energy change and the transition state theory. The newly established networks are termed as non-isothermal CRNs and shown to be efficient in modeling a broad class of non-isothermal chemical reaction systems. Moreover, we introduce detailed balanced networks for non-isothermal CRNs and provide them with a compact dynamic formula that exhibits at the same time the network topology and thermodynamic information. With this compact formula, the Legendre transformation, and some mild conditions, we show non-isothermal detailed balanced network systems to admit some fundamental properties,

1. the dissipativeness with respect to $S_{\mathcal{A}}(U, N)$ (Theorem 3.5),

2. the detailed balancing of each equilibrium (Corollary 3.6),

3. the existence, uniqueness, and asymptotic stability of the detailed balanced equilibrium (Theorem 4.5),

which are well consistent with results in isothermal detailed balanced networks. In contrast with the corresponding results for isothermal cases, the above properties require more conditions for non-isothermal detailed balanced networks. Specifically, (B.3) ensures the well posedness of thermodynamic quantities, Condition 1, Condition 2 and Condition 3 enable the non-isothermal CRNs to model a broad class of practical reaction systems, Condition 4 helps to construct a compacted formula for non-isothermal detailed balanced networks, and Condition 5 is used to prove the asymptotic stability by guaranteeing the modified Legendre transformation to be unbounded at any boundary. In a practical system, these conditions are usually easy to verify and, therefore, not too restrictive. In general, the analysis and results of this work, especially thermodynamic interpretations, provide insights into the research of non-isothermal chemical reaction systems.

There are numerous topics that we are pursuing, related to non-isothermal CRNT. First, for non-isothermal detailed balanced networks, it remains an open problem to extend the established locally asymptotic stability to a global result. Some methods that succeed in investigating the persistence of isothermal CRNs, such as strongly endotactic networks [24], the tier structure [3,5], semi-locking sets [4], and the toric differential inclusion $[14,24]$ can be helpful to this problem. Second, we will also investigate the connection between the network topology and unique/multi-stationarity, 
which can be applied to chemical engineering designs to preclude undesired stationary states. We suspect that the deficiency theory can still work for the equilibrium analysis of non-isothermal CRNs after some necessary modifications. Third, since oscillations are common phenomena in chemical engineering, it is also interesting to investigate what causes oscillations in non-isothermal CRNs. Forth, we are also exploring the stochastic behaviors of non-isothermal CRNs when systems' scales are small and trying to extract thermodynamic knowledge from them. The recent work [18] is a good starting point for this problem. Finally, though most relevant topics of non-isothermal CRNs are in the field of chemical engineering, there are still some applications in biological studies. A most direct application is the network used by Wang et. al. [46] that takes place in a very common protein synthesis circuit in the cell of E. Coli in the large intestine of human beings. The following three reactions are included in this network:

$\mathrm{LuxR}+\mathrm{AHL} \rightleftharpoons \mathrm{LuxR}-\mathrm{AHL}$,

$2(\mathrm{LuxR}-\mathrm{AHL}) \rightleftharpoons(\mathrm{LuxR}-\mathrm{AHL})_{2}$,

$(\mathrm{LuxR}-\mathrm{AHL})_{2}+\mathrm{DNA} \rightleftharpoons \mathrm{DNA}-(\mathrm{LuxR}-\mathrm{AHL})_{2}$.

More details about the network may be found in that paper. Also, it can be used to design control laws for a cell culturing system, where the temperature needs to be accurately regulated.

Appendix A. Notations and terminologies. Here we introduce some notations and terminologies used in this paper.

Mathematical Notation:

$\mathbb{R}^{n}, \mathbb{R}_{\geq 0}^{n}, \mathbb{R}_{>0}^{n}: n$-dimensional real space, nonnegative and positive real space, respectively.

$x^{v_{i}}: x^{v_{i}}=\prod_{j=1}^{d} x_{j}^{v_{j i}}$, where $x, v_{\cdot i} \in \mathbb{R}^{d}$ and $0^{0}$ is defined to be 1.

$\operatorname{Exp}(x): \operatorname{Exp}(x)=\left(\exp \left(x_{1}\right), \exp \left(x_{2}\right), \cdots, \exp \left(x_{d}\right)\right)^{\top}$ where $x \in \mathbb{R}_{>0}^{d}$.

$\operatorname{Ln}(x): \operatorname{Ln}(x)=\left(\ln x_{1}, \ln x_{2}, \cdots, \ln x_{d}\right)^{\top}$, where $x \in \mathbb{R}_{>0}^{d}$.

$\otimes$ : Cartesian product.

$\mathcal{I}_{n}:$ an $n \times n$ identity matrix.

$\mathbb{D}_{n}:$ an $n$-dimensional vector with every entry to be zero.

$\mathbb{1}_{n}:$ an $n$-dimensional vector with every entry to be one.

$\delta_{i}:$ an $n$-dimensional vector with the $i$-th component being 1 and the others zeros. $\chi(\cdot)$ : the indicator function.

\section{Physics terminologies:}

Activated state: the state corresponding to the highest potential energy along this reaction coordinate.

Callen's first postulate: a well mixed system can be fully described by internal energy, system volume, and mass amounts of involved substances.

Enthalpy: the sum of internal energy of a thermodynamic system and the work required to achieve its pressure and volume.

First law of thermodynamics: known as Law of Conservation of Energy, that states the total energy of an isolated system is constant, and cannot be created or destroyed.

Gibbs energy of activation: the energy which must be provided to a chemical system with potential reactants to result in a chemical reaction.

Internal energy: the energy contained within the thermodynamic system that can 
be increased by introduction of matter, by heat, or by doing thermodynamic work on the system.

Isolated system: a thermodynamic system that cannot exchange either energy or matter with the environment.

Isothermal system: a system with the temperature remaining constant.

Open system: a thermodynamic system that has external interactions taking the form of energy or material transfers into or out of the system boundary.

Port-Hamiltonian system: Hamiltonian system that has input and out ports and satisfies Dirac structures.

Process system: a system that transforms raw material and energy into products.

Second law of thermodynamics: the entropy of any isolated system always increases.

Appendix B. Related basic knowledge. In this appendix, we present some basic knowledge on thermodynamics and isothermal CRNT.

B.1. Thermodynamics. We first review some basic knowledge on thermodynamics. Usually, there are two perspectives to study thermodynamics, the reversible pathway argument originated from Carnot [13] and the statistical mechanics' viewpoint introduced by Gibbs [23], Maxwell [32,33], and Boltzmann [11]. In this part, we review the thermodynamics from the viewpoint of statistical mechanics, mainly referring to Sethna's textbook [35]. Notably, we assume, in this paper, the system to have a fixed volume and, for simplicity, the volume to be unitary.

B.1.1. Thermodynamic potentials. First, we consider a closed system where $n$ kinds of ideal fluids are well mixed. In such a system, We denote amounts of these substances as $N_{1}, N_{2}, \ldots, N_{n}$ (with the unit of mole) and the temperature as $T$ (with the unit of Kelvin, $K$ ). According to the canonical ensemble (c.f. [35]), each molecule of $i$-th substance is behaving randomly with respect to the MaxwellBoltzmann distribution

$$
\mathbb{P}\left(d \omega_{i}\right)=\frac{e^{-\frac{H_{i}\left(\omega_{i}\right)}{R T}}}{Z_{i}(T)} d \omega_{i},
$$

where $\omega_{i}$ is the microscopic state of the molecule taking value in a metric space $\Omega_{i}$, the function $H_{i}(\cdot)$ is a non-negative function called the Hamiltonian, the parameter $R$ is the Boltzmann constant equaling to $1.38 \times 10^{-23} \mathrm{~J} / K$, and $Z_{i}(T)$ is the normalization factor (or called the partition function of the single molecule system) defined by

$$
Z_{i}(T) \triangleq \int_{\Omega_{i}} \mathrm{e}^{-\frac{H_{i}\left(\omega_{i}\right)}{R T}} \mathrm{~d} \omega_{i}
$$

Here, we further assume the Hamiltonian, $H_{i}(\cdot)$, to have at least linear growth, i.e.

$$
H_{i}\left(\omega_{i}\right) \geq \mathfrak{C} \text { on } \cdot\left\|\omega_{i}\right\| \text { for all } i=1, \ldots, n \text { and some positive constant } \mathfrak{C} \text { on, }
$$

so that the integral in (B.2) is convergent. The Maxwell-Boltzmann distribution (B.1) works for both classical mechanics and quantum mechanics. In classical mechanics, $\omega_{i}$ is the vector of positions and momentums of the molecule with values taken in a high dimensional Euclidean space (i.e. $\Omega_{i}=\mathbb{R}^{d}$ where $d$ is the dimension of the space). Whereas in quantum mechanics, $\omega_{i}$ is a wave function representing the probability 
distribution of positions and momentums of the molecule, and the topology of the space $\Omega_{i}$ is much more complicated.

According to the Maxwell-Boltzmann distribution, each molecule has the average energy (with the unit of joule per mole $J / \mathrm{mol}$ )

$$
u_{i}(T) \triangleq A_{v} \times \frac{\int_{\Omega} H_{i}\left(\omega_{i}\right) \exp \left\{-\frac{H_{i}(\omega)}{R T}\right\} \mathrm{d} \omega_{i}}{Z_{i}(T)}
$$

where the integral is convergent due to (B.3), and the whole system has the average internal energy (with the unit of $J$ )

$$
U(T, N) \triangleq \sum_{i=1}^{n} N_{i} u_{i}(T)=N^{\top} u(T),
$$

where $A_{v}$ is the Avogadro number (a constant), $N=\left(N_{1}, N_{2}, \ldots, N_{n}\right)^{\top}$, and $u(T) \triangleq$ $\left(u_{1}(T), u_{2}(T), \cdots, u_{n}(T)\right)^{\top}$. The system's internal energy is the sum of the energy of each molecule in the system and, therefore, fluctuates over time due to the randomness of each molecule. However, by the law of large numbers, the fluctuation of the internal energy is extremely small compared to the average of the internal energy, and, therefore, from the viewpoint of statistics, the internal energy can be expressed by $U(T, N)$, a deterministic function of the temperature and mass amounts.

In statistic mechanics, the partition function is another important concept worth reviewing. For each molecular system, the partition function is defined by (B.2). For the whole system, the partition function is established based on the ones of molecular systems and has the expression

$$
Z(T, N) \triangleq \prod_{i=1}^{n} \frac{\left(Z_{i}(T)\right)^{A_{v} N_{i}}}{\left(A_{v} N_{i}\right) !},
$$

where the factorial term in the denominator is due to the indistinguishability of particles of each substance [35]. Particularly, the functions $Z_{i}(T)$ and $Z(T, N)$ are both non-descending with respect to the temperature component.

Proposition B.1. If the inequality (B.3) holds, then Partition functions, $Z_{i}(T)$ and $Z(T, N)$, are non-decreasing with respect to the temperature component.

Proof. Note that by definition $Z(T, N)$ is non-descending with respect to the temperature if each $Z_{i}(T)$ is non-descending. Therefore, we only need to show $Z_{i}(T)$ $(i=1, \ldots, n)$ to be non-decreasing. The derivative of $Z_{i}(T)$ is given by

$$
\frac{\mathrm{d} Z_{i}(T)}{\mathrm{d} T}=\int_{\Omega_{i}} \frac{H_{i}(\omega)}{R T^{2}} e^{-\frac{H_{i}\left(\omega_{i}\right)}{R T}} \mathrm{~d} \omega_{i} \geq 0,
$$

where the convergence of the integral is guaranteed by (B.3). Therefore, $Z_{i}(T)$ is non-descending, which proves the result.

Moreover, in this paper, we adopt the following assumption, which suggests each partition function $Z_{i}(T)$ to grow to infinity at the infinite temperature.

Condition 5. Each partition function $Z_{i}(T)$ satisfies $\lim _{T \rightarrow \infty} Z_{j}(T)=\infty$.

This assumption is not very restrictive as it holds true in many well-known cases, such as the ideal gas molecules where the Hamiltonian has a quadratic form, and, therefore, its partition function $Z_{i}(T)$ goes to infinity [35]. Condition 5 is mainly 
applied to showing the existence and uniqueness of a detailed balanced equilibrium, (see section 4 and Appendix C.2).

For the $i$-th species, each molecular system's Helmholtz free energy and entropy are given by

$$
g_{i}(T) \triangleq-A_{v} R T \ln Z_{i}(T) \quad \text { and } \quad s_{i}(T) \triangleq-\frac{\mathrm{d} g_{i}(T)}{\mathrm{d} T}
$$

with the units of $J / \mathrm{mol}$ and $J /($ mole $\cdot K)$, respectively [35]. For simplicity, we denote $g(T) \triangleq\left(g_{1}(T), g_{2}(T), \ldots, g_{n}(T)\right)^{\top}$ and $s(T) \triangleq\left(s_{1}(T), s_{2}(T), \ldots, s_{n}(T)\right)^{\top}$ in the context of this paper. Through simple calculation, one can arrive at the following expression

$$
s_{i}(T)=\frac{u_{i}(T)-g_{i}(T)}{T}
$$

which will be frequently used in later analyses. Moreover, according to L'Hospital's rule, the Helmholtz free energy satisfies the low temperature limit

$$
\lim _{T \rightarrow 0} g_{i}(T)=u_{i}(0)
$$

For the whole system, the Helmholtz free energy (with the unit of $J$ ) is defined by

$$
\begin{aligned}
G(T, N) & \triangleq-R T \ln Z(T, N) \\
& =\sum_{i=1}^{n}\left[\left(g_{i}(T)-A_{v} R T\right) N_{i}+A_{v} R T N_{i} \ln N_{i}+\mathcal{O}\left(R T \ln \left(A_{v} N_{i}\right)\right)\right],
\end{aligned}
$$

where $\mathcal{O}\left(R T \ln \left(A_{v} N_{i}\right)\right)$ represents a higher order term whose order of magnitude is at most the same as the one of $R T \ln \left(A_{v} N_{i}\right)$, and the second equality follows immediately from Stirling's approximation [35]. Since the term $R T \ln \left(A_{v} N_{i}\right)$ is very small in the molar scale and at the real-world temperature, the higher order term in the above formula can be neglected, and, therefore, the free energy is expressed as

$$
G(T, N) \triangleq \sum_{i=1}^{n}\left[\left(g_{i}(T)-A_{v} R T\right) N_{i}+A_{v} R T N_{i} \ln N_{i}\right] .
$$

Moreover, the whole system's entropy (with the unit of $J \cdot \operatorname{mole} / K$ ) is defined by

$$
S(T, N) \triangleq-\frac{\partial G(T, N)}{\partial T}=\sum_{i=1}^{N}\left[\left(s_{i}(T)+A_{v} R\right) N_{i}-A_{v} R N_{i} \ln N_{i}\right],
$$

and, by (B.7), (B.9), and (B.10), there holds the relation

$$
S(T, N)=\frac{U(T, N)-G(T, N)}{T} .
$$

B.1.2. Heat capacity and the fundamental equation. The heat capacity is the amount of heat supplying to a system to raise the temperature by a unit degree. For the molecule system (of $i$-th substance) and the whole system, the heat capacity is defined respectively as

$$
c_{i}(T) \triangleq \frac{\mathrm{d} u_{i}(T)}{\mathrm{d} T} \quad \text { and } \quad C(T, N) \triangleq \frac{\partial U(T, N)}{\partial T}=N^{\top} c(T),
$$


where $c(T)=\left(c_{1}(T), \ldots, c_{n}(T)\right)^{\top}$. Since the heat capacity of each molecule system can be seen as the variance of the Hamiltonian with respect to Boltzmann distribution [35], heat capacities, $c_{i}(T)$ and $C(T, N)$, are both non-negative functions. Moreover, by the at least linear growth of each Hamiltonian, the heat capacity $c_{i}(T)$ is strictly positive at any positive temperature.

Proposition B.2. If the inequality (B.3) holds, then

1. $c_{j}(T)>0$ for any $T>0$ and $i=1, \ldots, n$,

2. $C(T, N)>0$ for any $T>0$ and $N \in \mathbb{R}_{>0}^{n}$.

Proof. The inequality (B.3) suggests the function $H_{i}(\cdot)$ to be not an almost everywhere constant function, and, therefore, the heat capacity $c_{j}(T)$, the variance of $H_{i}(\cdot)$ with respect to the Maxwell-Boltzmann distribution, is great than 0 at any positive temperature. Therefore, the first result is shown. The second result follows immediately from the first result and the definition (B.12).

For a thermodynamic system with $n$ components, Callen's first postulate [12] states that $n+2$ extensive variables, $\left(U, V, N_{1}, \ldots, N_{n}\right)$ with $V$ the volume, define all the macroscopic properties of the system. Recall that we fixed the system's volume in this paper. Therefore, Callen's first postulate suggests the system we consider can be fully characterized by $n+1$ extensive variables, $\left(U, N_{1}, \ldots, N_{n}\right)$. The relations between other thermodynamic quantities (for instance, the temperature and the entropy) and these $n+1$ extensive variables are shown in the following.

Proposition B.3. If the inequality (B.3) holds, then for any

$$
(U, N)^{\top} \in\left\{(U, N)^{\top} \mid U>N^{\top} u(0)\right\} \bigcap \mathbb{R}_{>0}^{n+1}
$$

there hold

$$
d T=\frac{1}{C(T)} d U-\frac{u(T)}{C(T)} d N
$$

and

$$
d S=\frac{1}{T} d U-\frac{\mu(U, N)}{T} d N,
$$

where $\mu(U, N)=g(T)+A_{v} R T L n N$.

Proof. According to Proposition B.2, we have $\frac{\partial U(T, N)}{\partial T}=C(T, N)>0$ for all $(T, N)^{\top} \in \mathbb{R}_{>0}^{n+1}$. Thus by the implicit function theorem, the relation (B.13) follows immediately from differentiating (B.5) with the definition region $\left\{(U, N)^{\top} \mid U>\right.$ $\left.N^{\top} u(0)\right\} \cap \mathbb{R}_{>0}^{n+1}$. Moreover, by differentiating (B.10), we have the relation

$$
\begin{aligned}
\mathrm{d} S & =N^{\top} \frac{\mathrm{d}[(u(T)-g(T)) / T]}{\mathrm{d} T} \mathrm{~d} T+\left[s(T)-A_{v} R \operatorname{Ln} N\right]^{\top} \mathrm{d} N \\
& =\frac{N^{\top} c(T)}{T} \mathrm{~d} T+\left[s(T)-A_{v} R \operatorname{Ln} N\right]^{\top} \mathrm{d} N,
\end{aligned}
$$

where the first line follows from (B.7). By plugging (B.13) into the last line, we arrive at (B.14).

The equation (B.14) is the fundamental thermodynamic relation when the volume is fixed, and the term $\mu$ in (B.14) is called the chemical potential, which determines the direction of reactions [30]. Moreover, from the relation (B.14), we can observe that the entropy function $S(U, N)$ is strictly concave. 
Proposition B.4. If the inequality (B.3) holds, then $S(U, N)$ is strictly concave in the region $\left\{(U, N)^{\top} \mid U>N^{\top} u(0)\right\} \bigcap \mathbb{R}_{>0}^{n+1}$.

Proof. By (B.14) and (B.13), we can calculate the Hessian matrix of $S(U, N)$ as

$$
\mathcal{H}(U, N) \triangleq\left(\begin{array}{cc}
\frac{\partial^{2} S}{\partial U^{2}} & \frac{\partial^{2} S}{\partial U \partial N} \\
\left(\frac{\partial^{2} S}{\partial U \partial N}\right)^{\top} & \frac{\partial^{2} S}{\partial N^{2}}
\end{array}\right)=\frac{-1}{T^{2} C(T)}\left(\begin{array}{cc}
1 & -u^{\top}(T) \\
-u(T) & \mathcal{D}(N)+u(T) u^{\top}(T)
\end{array}\right),
$$

where $\mathcal{D}(N)=A_{v} R \cdot \operatorname{diag}\left\{\ln N_{1}, \ln N_{2}, \ldots, \ln N_{n}\right\}$. Obviously, $\mathcal{D}(N)$ is a positive diagonal matrix. Moreover, by denoting the matrix

$$
\mathcal{P} \triangleq\left(\begin{array}{cc}
1 & -u(T) \\
& \mathcal{I}_{n}
\end{array}\right)
$$

we arrive at the relation

$$
\mathcal{H}(U, N)=-\frac{1}{T^{2} C(T)} \mathcal{P}^{T}\left(\begin{array}{ll}
1 & \\
& \mathcal{D}(N)
\end{array}\right) \mathcal{P}<0,
$$

which proves this proposition.

In the context of this paper, we call a state $(U, N)$ positive if $(U, N)^{\top} \in \mathbb{R}_{>0}^{n}$ and $U>N^{\top} u(0)$, i.e., the internal energy, mass amounts, and temperature are all positive.

B.2. Isothermal CRNT. We then review basic concepts and terminologies about isothermal CRN.

Consider an isothermal CRN system with $n$ substances which are well mixed and a unit volume. Based on Callen's first postulate [12], the system can be fully described by the amounts of $n$ substances, i.e., $N=\left(N_{1}, \ldots, N_{n}\right)$. Further, assume that there are $r$ reactions taking place among $n$ substances with the reaction scheme

$$
\alpha_{1, j} X_{1}+\cdots+\alpha_{n, j} X_{n} \stackrel{k_{j}}{\longrightarrow} \tilde{\alpha}_{1, j} X_{1}+\cdots+\tilde{\alpha}_{n, j} X_{n}, \quad j=1, \ldots, r,
$$

where $X_{i}$ represents the $i$-th species in the system, $\alpha_{i, j}$ and $\tilde{\alpha}_{i, j}$ are non-negative integers called stoichiometric coefficients, the integer vectors, $\left(\alpha_{1, j}, \ldots, \alpha_{n, j}\right)^{\top}$ and $\left(\tilde{\alpha}_{1, j}, \ldots, \tilde{\alpha}_{n, j}\right)^{\top}$, are termed as complexes, and $k_{j}$ are reaction rate constants. The isothermal CRNT $[20,22,26]$ views the above reaction scheme as a graph, where the linear combinations of species on both sides of the reactions are the vertexes, the reaction arrows are the edges, and reaction rate constants are weights associated with edges. Mathematically, the isothermal $\mathrm{CRN}$ is proposed by a quadruplet $(\mathcal{S}, \mathcal{C}, \mathcal{R}, \mathcal{K})$ with the definition as follows.

Definition B.5 (Isothermal CRN $[20,22,26]$ ). An isothermal CRN is quadruplet $(\mathcal{S}, \mathcal{C}, \mathcal{R}, \mathcal{K})$, in which

- $\mathcal{S} \triangleq\left\{X_{1}, \ldots, X_{n}\right\}$ is the species set representing the considered substances,

- $\mathcal{C} \triangleq\left\{y_{1}, \ldots, y_{m}\right\}$ is the complex set consisting of all distinguished complexes where $m$ is the size of the set.

- $\mathcal{R} \triangleq\left\{y_{\sigma_{1}} \rightarrow y_{\pi_{1}}, \ldots, y_{\sigma_{r}} \rightarrow y_{\pi_{r}}\right\}$ is the reaction set with $\sigma_{j}$ and $\pi_{j}(1 \leq j \leq r)$ being respectively the indexes of the substrate and product complexes of the $j$-th reaction. Particularly, for each reaction, $\sigma_{j}$ and $\pi_{j}$ should not be the same.

- $\mathcal{K} \triangleq\left(k_{1}, \ldots, k_{r}\right)$ is the kinetics class. 
Utilizing the graph theory, recent literature $[34,43,44]$ introduce the complex matrix, $Y \triangleq\left(y_{1}, \ldots, y_{m}\right) \in \mathbb{R}^{n \times m}$ whose $j$ th column corresponds to the $j$ th complex in $\mathcal{C}$, and the incidence matrix, $D \in \mathbb{R}^{m \times r}$ where

$$
D_{i, j} \triangleq\left\{\begin{array}{cc}
1, & i=\pi_{j}, \\
-1, & i=\sigma_{j}, \\
0, & \text { otherwise, }
\end{array} \quad i \in\{1, \ldots, m\} \text { and } j \in\{1, \ldots, r\} .\right.
$$

The former corresponds to the complexes (vertexes) while the latter shows linkages between these complexes (i.e., edges). They jointly define the stoichiometric matrix $\Gamma$ by $\Gamma \triangleq Y D$. When equipped with mass-action kinetics, the reaction rate, denoted by $v_{j}(\cdot)$ for the $j$ th reaction, may be evaluated according to the power law with respect to the concentrations of substances, i.e.,

$$
v_{j}(N)=k_{j} N^{y_{\sigma_{j}} 4}=k_{j} \operatorname{Exp}\left(y_{\sigma_{j}}^{\top} \ln N\right), \quad j=1, \ldots, r,
$$

Therefore, the dynamics of the system has the form

$$
\dot{N}=Y D v(N)=\Gamma v(N),
$$

where $v(N)=\left(v_{1}(N), \ldots, v_{r}(N)\right)$. From (B.18), one can observe that the increment of the state, $N(t)-N(0)$, where $t$ and 0 are the time argument, always belongs to the linear space $\operatorname{Im} \Gamma$, and, therefore, the state can only evolve in an invariant set $(N(0)+\operatorname{Im} \Gamma) \cap \mathbb{R}_{\geq 0}^{n}$. The isothermal CRNT terms the linear space $\operatorname{Im} \Gamma$ as the stoichiometric subspace, the invariant sets $(N(0)+\operatorname{Im} \Gamma) \cap \mathbb{R}_{\geq 0}^{n}$ as the stoichiometric compatibility class, and the positive part of the invariant set, $(N(0)+\operatorname{Im} \Gamma) \cap \mathbb{R}_{>0}^{n}$, as the positive stoichiometric compatibility class.

Notably, along with real chemical reactions, CRN can also model boundary fluxes by using zero complexes to represent environment (see [41, section 5]), which makes the theory very useful in both chemical engineering and biology applications.

A mass-action $\mathrm{CRN}(\mathcal{S}, \mathcal{C}, \mathcal{R}, \mathcal{K})$ is reversible if forward and backward reactions $y_{\sigma_{j}} \rightarrow y_{\pi_{j}}$ and $y_{\pi_{j}} \rightarrow y_{\sigma_{j}}$ come in pairs. Among all chemical reaction networks, detailed balanced networks are special ones, where the network is reversible, and at some equilibrium, reaction rates of each pair of reversible reactions are balanced. In isothermal CRNT, such equilibria that balance the forward and backward reactions are termed as detailed balanced equilibria. In the past few decades, a large body of literature has investigated detailed balanced networks; a summary of the fundamental properties of them is listed as follows.

Theorem B.6 ( $[22,41])$. Let a mass-action isothermal $C R N(\mathcal{S}, \mathcal{C}, \mathcal{R}, \mathcal{K})$ be detailed balanced and possess a positive detailed balanced equilibrium $N^{*}$. By denoting

$$
G_{\mathcal{A}}(N) \triangleq N^{\top}\left(L n N-L n N^{*}\right)-\mathbb{1}_{n}^{\top}\left(N-N^{*}\right),
$$

the following properties hold.

1. $G_{\mathcal{A}}(N)$ (as a function of $N$ ) serves as a Lyapunov function for the system; $\dot{G}_{\mathcal{A}}(N) \leq 0$ where the equality holds if and only if $\nabla G(N) \in \operatorname{ker}\left(\Gamma^{\perp}\right)$

2. Any positive equilibrium of the system is detailed balanced.

\footnotetext{
${ }^{4}$ In the usual expression of mass-action kinetics, the reaction rate is proportional to the product of concentrations. As we restrict the system volume to be fixed and unitary, the concentrations and amounts of substances are numerically the same, and, thus, (B.17) does not violate the usual expression.
} 
3. In each positive stoichiometric compatibility class, there exists only one equilibrium, and this equilibrium is locally asymptotically stable.

The Lyapunov function $G_{\mathcal{A}}(N)$ first introduced by Horn and Jackson [26] is called the pseudo-Helmholtz free energy because of the similarity between these two functions' expressions. Specifically,we can easily observe by (B.9) that

$$
A_{v} R T^{*} G_{\mathcal{A}}(N)=G\left(T^{*}, N\right)-\frac{\partial G\left(T^{*}, N^{*}\right)}{\partial N}\left(N-N^{*}\right)-G\left(T^{*}, N^{*}\right),
$$

where $T^{*}$ is the temperature of the isothermal system, $G\left(T^{*}, N\right)$ is the Helmholtz free energy (see (B.9)), and the term on the right-hand side of the equality is the availability function of the Helmholtz free energy with respect to mass amounts (c.f. [2]).

Appendix C. Proofs of the main results. We give the detailed proofs of our two main theorems, Theorem 3.5 and Theorem 4.5, in this appendix.

C.1. The proof of Theorem 3.5. We first introduce basic concepts and properties of Laplacian matrix.

Definition C.1. A matrix $L \in \mathcal{R}^{m \times m}$ is a balanced Laplacian matrix if i) all its diagonal elements are positive, ii) all its off-diagonal elements are non-positive, and iii) its column sums and row sums are zero, i,e, $\mathbb{1}_{m}^{\top} L=\mathbb{O}_{m}^{\top}$ and $L \mathbb{1}_{m}=\mathbb{O}_{m}$.

Proposition C.2. The matrices, $B_{\mathrm{CR}} K_{\mathrm{CR}}(T) B_{\mathrm{CR}}^{\top}$ and $B_{\mathrm{IO}} K_{\mathrm{IO}} B_{\mathrm{IO}}^{\top}$, are both balanced Laplacian matrices when $T>0$.

Proof. For $B_{\mathrm{CR}} K_{\mathrm{CR}}(T) B_{\mathrm{CR}}^{\top}$ where $T>0$, each diagonal element satisfies

$$
\left(B_{\mathrm{CR}} K_{\mathrm{CR}}(T) B_{\mathrm{CR}}^{\top}\right)_{i, i}=B_{i} \cdot K_{\mathrm{CR}}(T) B_{i}^{\top} .>0, \quad i=1, \ldots, m,
$$

where the inequality follows from the positive definiteness of $K_{\mathrm{CR}}(T)$, and each offdiagonal element $\left(B_{\mathrm{CR}} K_{\mathrm{CR}}(T) B_{\mathrm{CR}}^{\top}\right)_{i_{1}, i_{2}}\left(i_{1}, i_{2}=1, \ldots, m\right.$ and $\left.i_{1} \neq i_{2}\right)$ satisfies

$$
\left(B_{\mathrm{CR}} K_{\mathrm{CR}}(T) B_{\mathrm{CR}}^{\top}\right)_{i_{1}, i_{2}}=B_{i_{1}} \cdot K_{\mathrm{CR}}(T) B_{i_{2}}^{\top}=\sum_{j=1}^{r_{\mathrm{CR}} / 2} B_{i_{1} j} B_{i_{2} j}\left(K_{\mathrm{CR}}(T)\right)_{j, j} \leq 0,
$$

where the inequality follows from $B_{i_{1} j} B_{i_{2} j} \leq 0$ for $i_{1} \neq i_{2}$ (c.f. (3.3)). Therefore, the first two conditions in Definition C.1 hold. Moreover, by the definition of $B$, it follows the relation $\mathbb{1}_{m}^{\top} B=\mathbb{O}_{m}^{\top}$, and, therefore, $\mathbb{1}_{m}^{\top} B_{\mathrm{CR}} K_{\mathrm{CR}}(T) B_{\mathrm{CR}}^{\top}=\mathbb{O}_{m}^{\top}$ and $B_{\mathrm{CR}} K_{\mathrm{CR}}(T) B_{\mathrm{CR}}^{\top} \mathbb{1}_{m}=\mathbb{O}_{m}$, which suggests the third condition in Definition C.1 to hold. Given the analysis above, we can conclude that $B_{\mathrm{CR}} K_{\mathrm{CR}}(T) B_{\mathrm{CR}}^{\top}$ is a Laplacian matrix when $T>0$.

Similarly, we can prove the result for $B_{\mathrm{IO}} K_{\mathrm{IO}} B_{\mathrm{IO}}^{\top}$, which shows the proposition. $\square$

Proposition C.3. If $T>0$, then there hold $\operatorname{ker}\left(B_{\mathrm{CR}} K_{\mathrm{CR}}(T) B_{\mathrm{CR}}^{\top}\right)=\operatorname{ker} B_{\mathrm{CR}}^{\top}$ and $\operatorname{ker}\left(B_{\mathrm{IO}} K_{\mathrm{IO}} B_{\mathrm{IO}}^{\top}\right)=\operatorname{ker} B_{\mathrm{IO}}^{\top}$.

Proof. If $\gamma \in \operatorname{ker}\left(B_{\mathrm{CR}} K_{\mathrm{CR}}(T) B_{\mathrm{CR}}^{\top}\right)$, then $\gamma^{\top} \operatorname{ker} B_{\mathrm{CR}} K_{\mathrm{CR}}(T) B_{\mathrm{CR}}^{\top} \gamma=0$. By the positive definiteness of $K_{\mathrm{CR}}(T)$ when $T>0$, we have the relation $B_{\mathrm{CR}}^{\top} \gamma=\mathbb{O}_{r_{\mathrm{CR}}}$, which suggests $\operatorname{ker}\left(B_{\mathrm{CR}} K_{\mathrm{CR}}(T) B_{\mathrm{CR}}^{\top}\right) \subset \operatorname{ker}\left(B_{\mathrm{CR}}^{\top}\right)$. Since one can easily observe the relation $\operatorname{ker}\left(B_{\mathrm{CR}}^{\top}\right) \subset \operatorname{ker}\left(B_{\mathrm{CR}} K_{\mathrm{CR}}(T) B_{\mathrm{CR}}^{\top}\right)$, we therefore can conclude $\operatorname{ker}\left(B_{\mathrm{CR}}^{\top}\right)=$ $\operatorname{ker}\left(B_{\mathrm{CR}} K_{\mathrm{CR}}(T) B_{\mathrm{CR}}^{\top}\right)$.

Similarly, we can prove $\operatorname{ker}\left(B_{\mathrm{IO}} K_{\mathrm{IO}} B_{\mathrm{IO}}^{\top}\right)=\operatorname{ker}\left(B_{\mathrm{IO}}^{\top}\right)$, which shows the result. 
Lemma C.4 ([34,43]). If $L$ is a balanced Laplacian matrix, then $\gamma^{\top} L \operatorname{Exp}(\gamma) \geq 0$ for any $\gamma \in \mathbb{R}^{n}$, where the equality holds if $L^{\top} \gamma=0$.

Proposition C.5. If (B.3) and $r_{\mathrm{IO}} \neq 0$ hold, then each component of the vector $\left(\frac{1}{T^{*}}-\frac{1}{T}\right) \Delta \mathcal{U}_{\mathrm{IO}}+\left(\frac{g(T)}{T}-\frac{g\left(T^{*}\right)}{T^{*}}\right)^{\top} Y D_{\mathrm{IO}}$ is zero when $T=T^{*}$ and otherwise negative.

Proof. By denoting the $j$-th element of the considered vector as $\xi_{j}(T)$, we have

$$
\xi_{j}(T)=-u_{i}(T) / T^{*}+u_{i}(T) / T-g_{i}(T) / T+g_{i}\left(T^{*}\right) / T^{*}, \quad \text { for } y_{\sigma_{j+r_{\mathrm{CR}}}}=\delta_{i},
$$

and, by (3.1),

$$
\xi_{j}(T)=u_{i}\left(T^{*}\right) / T^{*}-u_{i}\left(T^{*}\right) / T+g_{i}(T) / T-g_{i}\left(T^{*}\right) / T^{*}, \quad \text { for } y_{\pi_{j+r} \mathrm{CR}}=\delta_{i} .
$$

According to above equations, we can observe that all $\xi_{j}(T)$ are zero when $T=T^{*}$. Moreover, by (B.7), we can easily calculate the derivative of $\xi_{j}(T)$ as

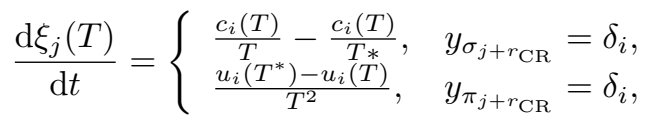

which suggests the derivative of $\xi_{j}$ to be zero at $T=T^{*}$, positive for $T<T^{*}$, and negative for $T>T^{*}$. Given the analysis above, we can conclude that each $\xi_{j}(T)$ is zero at $T=T^{*}$ and otherwise negative, which shows the result.

With these preparation, we can finally prove Theorem 3.5.

The proof of Theorem 3.5. According to the chain rule and the dynamics (3.11), it follows that

$$
\begin{aligned}
& \dot{S}_{\mathcal{A}}(U, N) \\
& =-\left(\frac{\mu}{T}-\frac{\mu^{*}}{T^{*}}\right)^{\top} Y B_{\mathrm{CR}} K_{\mathrm{CR}}(T) B_{\mathrm{CR}}^{\top} \operatorname{Exp}\left(Y\left(\frac{\mu}{A_{v} R T}-\frac{\mu^{*}}{A_{v} R T^{*}}\right)\right) \\
& \quad-A_{v} R\left(\operatorname{Ln} N-\operatorname{Ln} N^{*}\right)^{\top} Y B_{\mathrm{IO}} K_{\mathrm{IO}} B_{\mathrm{IO}}^{\top} \operatorname{Exp}\left(Y\left(\operatorname{Ln} N-\operatorname{Ln} N^{*}\right)\right) \\
& \quad+\left[\left(1 / T^{*}-1 / T\right) \Delta \mathcal{U}_{\mathrm{IO}}+\left(g(T) / T-g\left(T^{*}\right) / T^{*}\right)^{\top} Y D_{\mathrm{IO}}\right] v_{\mathrm{IO}} \\
& \quad+\left(1 / T^{*}-1 / T\right) \Delta \mathcal{U}_{\mathrm{CR}} v_{\mathrm{CR}}(U, N)-\tilde{k}_{r_{\mathrm{CR}}+r_{\mathrm{IO}}+1} h\left(T^{*}-T\right)^{2} \chi\left(r_{\mathrm{HE}} \neq 0\right) /\left(T^{*} T\right) \\
& \leq .1) \\
& \leq\left[\left(1 / T^{*}-1 / T\right) \Delta \mathcal{U}_{\mathrm{IO}}+\left(g(T) / T-g\left(T^{*}\right) / T^{*}\right)^{\top} Y D_{\mathrm{IO}}\right] v_{\mathrm{IO}} \\
& \quad+\left(1 / T^{*}-1 / T\right) \Delta \mathcal{U}_{\mathrm{CR}} v_{\mathrm{CR}}(U, N)-\tilde{k}_{r_{\mathrm{CR}}+r_{\mathrm{IO}}+1} h\left(T^{*}-T\right)^{2} \chi\left(r_{\mathrm{HE}} \neq 0\right) /\left(T^{*} T\right),
\end{aligned}
$$

where the inequality follows from Proposition C.2 and Lemma C.4, and the equality holds if and only if $\left(\mu / T-\mu^{*} / T^{*}\right) \in \operatorname{ker}\left(B_{\mathrm{CR}}^{\top} Y^{\top}\right)$ and $\left(\operatorname{Ln} N-\operatorname{Ln} N^{*}\right) \in \operatorname{ker}\left(B_{\mathrm{IO}}^{\top} Y^{\top}\right)$ (by Lemma C.4 and Proposition C.3). According to Condition 2, there are several cases, we need to analyze separately:

1. For $\Delta U_{\mathrm{CR}} \neq \mathbb{O}_{r_{\mathrm{CR}}}$ and $r_{\mathrm{CR}}=r_{\mathrm{IO}}=0$ (i.e. the second case in Condition 2), the inequality (C.1) suggests that $\dot{S}_{\mathcal{A}}(U, N) \leq 0$ at any positive state with $T=T^{*}$ where the inequality holds when $\left(\mu / T-\mu^{*} / T^{*}\right) \in \operatorname{ker}\left(B_{\mathrm{CR}}^{\top} Y^{\top}\right)$. Since $\operatorname{ker}\left(B_{\mathrm{CR}}^{\top} Y^{\top}\right)=\operatorname{ker}\left(B^{\top} Y^{\top}\right)=\operatorname{ker}\left(\Gamma^{\top}\right)$ (by Proposition 3.4) hold in this case, we can further conclude $\dot{S}_{\mathcal{A}}(U, N) \leq 0$ at any positive state with $T=T^{*}$ where the inequality holds if and only if $\nabla S_{\mathcal{A}}(U, N) \in \operatorname{ker} \tilde{\Gamma}^{\top}$. 
2. For $\Delta U_{\mathrm{CR}}=\mathbb{O}_{r_{\mathrm{CR}}}$ and $r_{\mathrm{CR}}=r_{\mathrm{IO}}=0$ (i.e. a part of the first case in Condition 2), the inequality (C.1) suggests that $\dot{S}_{\mathcal{A}}(U, N) \leq 0$ at any positive state where the equality holds if and only if $\left(\mu / T-\mu^{*} / T^{*}\right) \in \operatorname{ker}\left(B_{\mathrm{CR}}^{\top} Y^{\top}\right)$. Since $\operatorname{ker}\left(B_{\mathrm{CR}}^{\top} Y^{\top}\right)=\operatorname{ker}\left(B^{\top} Y^{\top}\right)=\operatorname{ker}\left(\Gamma^{\top}\right)$ (by Proposition 3.4) hold in this case, we can further conclude $\dot{S}_{\mathcal{A}}(U, N) \leq 0$ at any positive state where the inequality holds if and only if $\nabla S_{\mathcal{A}}(U, N) \in \operatorname{ker}\left(\tilde{\Gamma}^{\top}\right)$.

3. For $\Delta U_{\mathrm{CR}}=\mathbb{O}_{r_{\mathrm{CR}}}$ and at least one of $r_{\mathrm{CR}}$ and $r_{\mathrm{IO}}$ being non-zero (i.e. the other part of the first case in Condition 2), the inequality (C.1) suggests by Proposition C.5 that $\dot{S}_{\mathcal{A}}(U, N) \leq 0$ at any positive state where the equality hold if and only if $\left(\mu / T-\mu^{*} / T^{*}\right) \in \operatorname{ker}\left(B_{\mathrm{CR}}^{\top} Y^{\top}\right),(\operatorname{Ln} N-$ $\left.\operatorname{Ln} N^{*}\right) \in \operatorname{ker}\left(B_{\mathrm{IO}}^{\top} Y^{\top}\right)$ and $T=T^{*}$. Note that the condition $\left(\operatorname{Ln} N-\operatorname{Ln} N^{*}\right) \in$ $\operatorname{ker}\left(Y B_{\mathrm{IO}}\right)$ and $T=T^{*}$ suggests $\left(\mu / T-\mu^{*} / T^{*}\right)\left(=g(T) / T-g\left(T^{*}\right) / T^{*}+\right.$ $\left.A_{v} R\left(\operatorname{Ln} N-\operatorname{Ln} N^{*}\right)\right)$ to be in the space $\operatorname{ker}\left(B_{\mathrm{IO}}^{\top} Y^{\top}\right)$. Therefor, we can further conclude that $\dot{S}_{\mathcal{A}}(U, N) \leq 0$ where the the equality holds if and only if $\frac{\partial S_{\mathcal{A}}(U, N)}{\partial N} \in \operatorname{ker}\left(B_{\mathrm{CR}}^{\top} Y^{\top}\right)+\operatorname{ker}\left(B_{\mathrm{IO}}^{\top} Y^{\top}\right)=\operatorname{ker}\left(B^{\top} Y^{\top}\right)=\operatorname{ker}\left(\Gamma^{\top}\right)$ and $\frac{\partial S_{\mathcal{A}}(U, N)}{\partial U}=0$ (i.e. $\left.\nabla S_{\mathcal{A}}(U, N) \in \operatorname{ker}\left(\tilde{\Gamma}^{\top}\right)\right)$.

Given the analysis above, we show the function $S_{\mathcal{A}}(U, N)$ to be dissipative.

Note that, besides dissipativeness, $S_{\mathcal{A}}(U, N)$ is also positive definite in the positive stoichiometric-like compatibility class $\mathcal{P S}\left(U^{*}, N^{*}\right)$. Therefore, by Lyapunov's second method, the function $S_{\mathcal{A}}(U, N)$ is a Lyapunov function for the dynamics (3.11), rendering the state $\left(U^{*}, N^{*}\right)$ to be stable.

C.2. The proofs of Theorem 4.5. In this subsetion, we provide the proofs for results related to Theorem 4.5.

Proof of Proposition 4.1. We first show the unboundedness of $\mathcal{L}_{A}(\beta, \gamma)$ at any finite boundary, i.e. (4.4). According to the definition, we can re-express $\mathcal{L}_{A}(\beta, \gamma)$ as

$$
\begin{aligned}
\mathcal{L}_{A}(\beta, \gamma) & =S(U, N)+\left(\beta-\frac{1}{T^{*}}\right) U+\left(\gamma+\frac{\mu^{*}}{T^{*}}\right)^{\top} N-\beta U^{o}-\gamma^{\top} N^{o}+\mathfrak{C o n} \\
& =S(U, N)-\frac{U}{T}+\frac{G}{T}+A_{v} R \sum_{i=1}^{n} N_{i}-\beta U^{o}-\gamma^{\top} N^{o}+\mathfrak{C o n} \\
& =A_{v} R\left(\sum_{i=1}^{n} N_{i}\right)-\beta U^{o}-\gamma^{\top} N^{o}+\mathfrak{C o n}
\end{aligned}
$$

where $\mathfrak{C}$ on is some constant, and the last equality follows from (B.11). By (4.2), we can conclude that $\lim _{\beta \rightarrow\left(\frac{1}{T^{*}}\right)^{-}} T=+\infty$, and, therefore,

$$
\lim _{(\beta, \gamma) \rightarrow\left(\beta^{b^{-}}, \gamma^{b}\right)} N_{i}=\lim _{T \rightarrow \infty} Z_{i}(T) \exp \left(\frac{\gamma^{b}+\frac{\mu^{*}}{T^{*}}}{A_{v} R}\right)=+\infty, \quad \forall\left(\beta^{b}, \gamma^{b}\right) \in\left\{\frac{1}{T^{*}}\right\} \otimes \mathbb{R}^{n},
$$

where the first equality follows from (4.3), and the second from Condition 5. By plugging it into (C.2), we arrive at (4.4), i.e. the unboundedness of $\mathcal{L}_{A}(\beta, \gamma)$ at any finite boundary. 
Then, we show part of (4.5) where $\bar{\beta}=0$ and $\bar{\gamma} \neq \mathbb{O}_{n}$. In this case, we obtain

$$
\begin{aligned}
& \lim _{\theta \rightarrow+\infty} \mathcal{L}_{\mathcal{A}}(\theta \bar{\beta}, \theta \bar{\gamma})= \mathcal{L}_{\mathcal{A}}\left(0, \mathbb{D}_{n}\right)+\lim _{\theta \rightarrow+\infty} \int_{0}^{\theta} \bar{\gamma}^{\top}\left(N-N^{o}\right) \mathrm{d} \eta \\
&= \mathcal{L}_{\mathcal{A}}\left(0, \mathbb{D}_{n}\right)+\lim _{\theta \rightarrow+\infty} \int_{0}^{\theta} \bar{\gamma}^{\top}\left(\operatorname{Exp}\left\{\frac{\left.\left.\eta \bar{\gamma}+\frac{\mu^{*}}{T^{*}}-\frac{g_{i}(T *)}{T *}\right\}-N^{o}\right) \mathrm{d} \eta}{A_{v} R}\right\}\right. \\
&=\mathcal{L}_{\mathcal{A}}\left(0, \mathbb{O}_{n}\right)+\lim _{\theta \rightarrow+\infty}\left(A_{v} R \bar{\gamma}^{\top} \operatorname{Exp}\left\{\frac{\theta \bar{\gamma}+\frac{\mu^{*}}{T^{*}}-\frac{g_{i}(T *)}{T *}}{A_{v} R}\right\}-\theta \bar{\gamma}^{\top} N^{o}\right. \\
&\left.-A_{v} R \bar{\gamma}^{\top} \operatorname{Exp}\left\{\frac{\frac{\mu^{*}}{T^{*}}-\frac{g_{i}(T)}{T}}{A_{v} R}\right\}\right) \\
&=+\infty,
\end{aligned}
$$

where the second equality follows from (4.2) and (4.3), and the last equality follows from the unboundedness of function $f(x)=b_{1} e^{a x}-a b x,\left(b_{1}, b_{2}>0, a \neq 0\right)$, at infinities.

Finally, we show the rest part of $(4.5)$ where $\bar{\beta}<0$. In this case, we can calculate

$$
\begin{aligned}
\frac{\mathcal{L}_{\mathcal{A}}(\theta \bar{\beta}, \theta \bar{\gamma})}{\mathrm{d} \theta} & =\bar{\beta}\left(U-U^{o}\right)+\bar{\gamma}^{\top}\left(N-N^{o}\right) \\
& =-\bar{\beta}\left[U^{o}-\left(N^{o}\right)^{\top} u(0)\right]+N_{i}\left(u_{i}(T) \bar{\beta}_{i}+\bar{\gamma}_{i}\right)-N_{i}^{o}\left(u_{i}(0) \bar{\beta}_{i}+\bar{\gamma}_{i}\right) .
\end{aligned}
$$

We further denote $\operatorname{term}_{i}(\theta \bar{\beta}, \theta \bar{\gamma}) \triangleq N_{i}\left(u_{i}(T) \bar{\beta}_{i}+\bar{\gamma}_{i}\right)-N_{i}^{o}\left(u_{i}(0) \bar{\beta}_{i}+\bar{\gamma}_{i}\right)$ and, by (4.2) and (4.3), arrive at

$$
\begin{aligned}
& \lim _{\theta \rightarrow \infty} \operatorname{term}_{i}(\theta \bar{\beta}, \theta \bar{\gamma}) \\
& =\lim _{\theta \rightarrow \infty} \exp \left(\frac{\left.\theta \bar{\gamma}_{i}+\frac{\mu^{*}}{T^{*}}-\frac{g_{i}(T)}{T}\right)\left(u_{i}(T) \bar{\beta}_{i}+\bar{\gamma}_{i}\right)-N_{i}^{o}\left(u_{i}(0) \bar{\beta}_{i}+\bar{\gamma}_{i}\right)}{A_{v}}\right) \\
& =\lim _{\theta \rightarrow \infty} \exp \left(\frac{\theta\left(\bar{\gamma}_{i}+u_{i}(0) \bar{\beta}\right)+\frac{\mu^{*}}{T^{*}}}{A_{v} R}\right)\left(u_{i}(0) \bar{\beta}_{i}+\bar{\gamma}_{i}\right)-N_{i}^{o}\left(u_{i}(0) \bar{\beta}_{i}+\bar{\gamma}_{i}\right),
\end{aligned}
$$

where the second equality follows from $\lim _{\theta \rightarrow \infty} T=0, \lim _{\theta \rightarrow \infty} 1 / T=\lim _{\theta \rightarrow \infty} \bar{\beta} \theta$, and (B.8). To calculate the limit of $\operatorname{term}_{i}(\theta \bar{\beta}, \theta \bar{\gamma})$, we need to consider three cases:

1. " $u_{i}(0) \bar{\beta}_{i}+\bar{\gamma}_{i}>0$ ": In this case, the exponential term in the above dominants, and therefore, $\lim _{\theta \rightarrow \infty} \operatorname{term}_{i}(\theta \bar{\beta}, \theta \bar{\gamma})=\infty$.

2. " $u_{i}(0) \bar{\beta}_{i}+\bar{\gamma}_{i}=0$ ": In this case, $\operatorname{term}_{i}(\theta \bar{\beta}, \theta \bar{\gamma}) \equiv 0$.

3. " $u_{i}(0) \bar{\beta}_{i}+\bar{\gamma}_{i}<0$ ": In this case, the constant term in the above dominants, and therefore, $\lim _{\theta \rightarrow \infty} \operatorname{term}_{i}(\theta \bar{\beta}, \theta \bar{\gamma})>0$.

To conclude, there exists a positive value $\bar{\theta}$ such that for any $\theta>\bar{\theta}$ each term $_{i}(\theta \bar{\beta}, \theta \bar{\gamma})>$ 0 , and, therefore

$$
\frac{\mathcal{L}_{\mathcal{A}}(\theta \bar{\beta}, \theta \bar{\gamma})}{\mathrm{d} \theta}>-\bar{\beta}\left[U^{o}-\left(N^{o}\right)^{\top} u(0)\right], \quad \forall \theta>\bar{\theta} .
$$

This fact suggests $\mathcal{L}_{\mathcal{A}}(\theta \bar{\beta}, \theta \bar{\gamma})$ to have at least linear growth with respect to $\theta$ and go to infinity as $\theta \rightarrow+\infty$.

Given the analysis above, we prove the proposition. 
Proof of Proposition 4.2. The non-emptiness of $\mathfrak{S}_{c}$ follows from $\left(0, \mathbb{O}_{n}\right) \in \mathfrak{S}_{c}$; the convexity follows from the convexity of the function $\mathcal{L}_{A}(\beta, \gamma)$. Therefore, we only need to show the closeness of this set. Let $\left\{\left(\beta^{\ell}, \gamma^{\ell}\right)\right\}_{\ell}$ be a convergent sequence in $\mathfrak{S}_{c}$ with the limit $\left(\beta^{\infty}, \gamma^{\infty}\right)$ (in the closure of $\mathfrak{D} \cap \operatorname{ker}\left(\tilde{\Gamma}^{\top}\right)$ ). By (4.4), we know that $\left(\beta^{\infty}, \gamma^{\infty}\right)$ cannot be on the boundary of $\mathfrak{D}$ and, therefore, must in the interior of $\mathfrak{D} \cap \operatorname{ker}\left(\tilde{\Gamma}^{\top}\right)$. Moreover, by the continuity, we have $\mathcal{L}_{A}\left(\beta^{\infty}, \gamma^{\infty}\right)=\lim _{\ell \rightarrow \infty} \mathcal{L}_{A}\left(\beta^{\ell}, \gamma^{\ell}\right) \leq \mathcal{L}_{A}\left(0, \mathbb{O}_{n}\right)$, which suggests $\left(\beta^{\infty}, \gamma^{\infty}\right)$ to be in the set $\mathfrak{S}_{c}$. Therefore, we show the result.

Proof of Proposition 4.3. We first prove by contradiction that $\mathfrak{S}_{c}$ contains no half line with endpoint $\mathbb{D}_{n+1}$. Assume $\mathfrak{S}_{c}$ contains a half line $\{(\theta \bar{\beta}, \theta \bar{\gamma}) \mid \theta>0\}$ where $(\bar{\beta}, \bar{\gamma})^{\top} \neq \mathbb{O}_{n+1}$. By $(4.5)$ and the definition of $\mathfrak{S}_{c}$, the parameter $\bar{\beta}$ should be greater than zero; by the fact $\mathfrak{D}=\left(-\infty, \frac{1}{T^{*}}\right) \otimes \mathbb{R}^{n}$ is only half of $\mathbb{R}^{n+1}$, the parameter $\bar{\beta}$ cannot be greater than zero. As a result, $\mathfrak{S}_{c}$ contains no half line with endpoint $\mathbb{O}_{n+1}$.

Note that an unbounded closed and convex set containing $\mathbb{O}_{n+1}$ must have a half line with endpoint $\mathbb{O}_{n+1}\left[38\right.$, P. 105]. Therefore, the closed convex set $\mathfrak{S}_{c}$ (c.f. Proposition 4.2) is bounded, and, moreover, compact.

Proof of Proposition 4.4. The compactness of $\mathfrak{S}_{c}$ (c.f. Proposition 4.3) suggests the continuous function $\mathcal{L}_{A}(\beta, \gamma)$ to have a locally minimum point in the region $\mathfrak{S}_{c}$. Moreover, by the definition of $\mathfrak{S}_{c}$, this locally minimum value is less than any value $\mathcal{L}_{A}(\beta, \gamma)$ takes outside $\mathfrak{S}_{c}$. Therefore, this minimum point is also a minimum in $\mathfrak{D} \cap \operatorname{ker}\left(\tilde{\Gamma}^{\top}\right)$.

The uniqueness of the minimum point follows immediately from the strict convexity of $\mathcal{L}_{A}(\beta, \gamma)$, and, therefore, we prove the result.

Proof of Theorem 4.5. Existence: Let $\left(\beta^{* *}, \gamma^{* *}\right)$ be the minimum point of $\mathcal{L}_{A}(\beta, \gamma)$ in the region $\mathfrak{D} \cap \operatorname{ker}\left(\tilde{\Gamma}^{\top}\right)$, where the existence of this point is guaranteed by Proposition 4.4. By denoting $U^{* *} \triangleq \frac{\partial \mathcal{L}\left(\beta^{* *}, \gamma^{* *}\right)}{\partial \beta}=\frac{\partial \mathcal{L}_{\mathcal{A}}\left(\beta^{* *}, \gamma^{* *}\right)}{\partial \beta}+U^{o}$ and $N^{* *} \triangleq\left(\frac{\partial \mathcal{L}\left(\beta^{* *}, \gamma^{* *}\right)}{\partial \gamma}\right)^{\top}=\left(\frac{\partial \mathcal{L}_{\mathcal{A}}\left(\beta^{* *}, \gamma^{* *}\right)}{\partial \beta}\right)^{\top}+N^{o}$, we can conclude that $\left(U^{* *}, N^{* *}\right)$ is a positive state (by the definition of the Legendre transformation) and satisfies (by the minimum of $\left.\left(\beta^{* *}, \gamma^{* *}\right)\right)$

$$
\left(\begin{array}{c}
U^{* *}-U^{o} \\
N^{* *}-N^{o}
\end{array}\right)=\nabla \mathcal{L}_{\mathcal{A}}\left(\beta^{* *}, \gamma^{* *}\right) \in \operatorname{Im} \tilde{\Gamma}
$$

which suggests $\left(U^{* *}, N^{* *}\right) \in \mathcal{P} \mathcal{S}\left(U^{o}, N^{o}\right)$. Note that if $\Delta \mathcal{U}_{\mathrm{CR}} \neq \mathbb{D}^{r} \mathrm{CR}$, then the condition $U^{o}=\left(N^{o}\right)^{\top} u\left(T_{e}\right)$, Condition 2, and (C.3) implies $U^{* *}=\left(N^{* *}\right)^{\top} u\left(T_{e}\right)$. According to the Legendre transformation $\mathcal{L}(\beta, \gamma)$, we can further conclude that

$$
\nabla S_{\mathcal{A}}\left(U^{* *}, N^{* *}\right)=\left(\begin{array}{c}
\beta^{* *} \\
\gamma^{* *}
\end{array}\right) \in \operatorname{ker}\left(\tilde{\Gamma}^{\top}\right)
$$

and, therefore, $\left(U^{* *}, N^{* *}\right)$ is a detailed balanced equilibrium in $\mathcal{P} \mathcal{S}\left(U^{o}, N^{o}\right)$ (by Corollary 3.6).

Uniqueness: Note that Corollary 3.6 suggests any positive detailed balanced equilibrium to be a stationary point of $S_{\mathcal{A}}(U, N)$ in its positive stoichiometric compatibility class. Therefore, the uniqueness of the detailed balanced equilibrium follows immediately from the strict convexity of $S_{\mathcal{A}}(U, N)$.

Asymptotic stability: As mentioned above, any positive detailed balanced equilibrium is a stationary point of $S_{\mathcal{A}}(U, N)$ in its positive stoichiometric compatibility class. Therefore, the strict convexity of $S_{\mathcal{A}}(U, N)$ suggests the function $S_{\mathcal{A}}(U, N)$ to be lower bounded in $\mathcal{P} \mathcal{S}\left(U^{o}, N^{o}\right)$ with the minimum point at the unique detailed balanced equilibrium. Note that by Theorem 3.5 and Corollary 3.6, the function 
$S_{\mathcal{A}}(U, N)$ is strictly dissipative at any positive state other than detailed balanced equilibrium. Thus, by the second Lyapunov method, the unique detailed balanced equilibrium is locally asymptotically stable.

\section{REFERENCES}

[1] M. A. Al-Radhawi And D. Angeli, New approach to the stability of chemical reaction networks: Piecewise linear in rates lyapunov functions, IEEE Transactions on Automatic Control, 61 (2016), pp. 76-89.

[2] A. A. Alonso And B. E. Ydstie, Stabilization of distributed systems using irreversible thermodynamics, Automatica, 37 (2001), pp. 1739-1755.

[3] D. Anderson, D. Cappelletti, J. Kim, And T. Nguyen, Tier structure of strongly endotactic reaction networks, arXiv preprint arXiv:1808.05328, (2018).

[4] D. F. Anderson, Global asymptotic stability for a class of nonlinear chemical equations, SIAM Journal on Applied Mathematics, 68 (2008), pp. 1464-1476.

[5] D. F. Anderson, A proof of the global attractor conjecture in the single linkage class case, SIAM Journal on Applied Mathematics, 71 (2011), pp. 1487-1508.

[6] D. F. Anderson, G. Craciun, M. Gopalkrishnan, and C. Wiuf, Lyapunov functions, stationary distributions, and non-equilibrium potential for reaction networks, Bulletin of mathematical biology, 77 (2015), pp. 1744-1767.

[7] D. F. Anderson, G. Craciun, and T. G. Kurtz, Product-form stationary distributions for deficiency zero chemical reaction networks, Bulletin of mathematical biology, 72 (2010), pp. 1947-1970.

[8] P. Atkins and J. De Paula, Elements of physical chemistry, Oxford University Press, USA, 2013.

[9] D. A. Beard, E. Babson, E. Curtis, And H. Qian, Thermodynamic constraints for biochemical networks, Journal of theoretical biology, 228 (2004), pp. 327-333.

[10] D. A. BeARD AND H. QIAN, Thermodynamic-based computational profiling of cellular regulatory control in hepatocyte metabolism, American Journal of Physiology-Endocrinology and Metabolism, 288 (2005), pp. E633-E644.

[11] L. Boltzmann, Lectures on gas theory, Courier Corporation, 2012.

[12] H. B. CALlen, Thermodynamics and an introduction to thermostatistics, 1998.

[13] S. CARnot, Réflexions sur la puissance motrice de feu et sur les machines propresa développer cette puissance. paris: Bachelier 1824, Deutsche Übersetzung von W. Ostwald: Betrachtungen über die bewegende Kraft des Feuers und die zur Entwicklung dieser Kraft geeigneten Maschinen. Ostwalds Klassiker d. exakten Wissensch.

[14] G. CRaciun, Toric differential inclusions and a proof of the global attractor conjecture, arXiv preprint arXiv:1501.02860, (2015).

[15] G. Craciun and M. Feinberg, Multiple equilibria in complex chemical reaction networks: Ii. the species-reaction graph, SIAM Journal on Applied Mathematics, 66 (2006), pp. 13211338.

[16] H. Eyring, The activated complex in chemical reactions, The Journal of Chemical Physics, 3 (1935), pp. 107-115.

[17] Z. FANG AND C. GAO, Lyapunov function partial differential equations for chemical reaction networks: Some special cases, SIAM Journal on Applied Dynamical Systems, 18 (2019), pp. 1163-1199.

[18] Z. Fang, B. E. Ydstie, and C. GaO, Thermodynamic potentials from stationary probabilities, IFAC-PapersOnLine, 52 (2019), pp. 96-102.

[19] M. Feinberg, Complex balancing in general kinetic systems, Archive for rational mechanics and analysis, 49 (1972), pp. 187-194.

[20] M. Feinberg, Chemical reaction network structure and the stability of complex isothermal reactorsi. the deficiency zero and deficiency one theorems, Chemical Engineering Science, 42 (1987), pp. 2229-2268.

[21] M. FeinBerg, Chemical reaction network structure and the stability of complex isothermal reactorsii. multiple steady states for networks of deficiency one, Chemical Engineering Science, 43 (1988), pp. 1-25.

[22] M. FeInBERG, The existence and uniqueness of steady states for a class of chemical reaction networks, Archive for Rational Mechanics and Analysis, 132 (1995), pp. 311-370.

[23] J. W. GIBBS, On the equilibrium of heterogeneous substances, (1879).

[24] M. Gopalkrishnan, E. Miller, and A. Shiu, A geometric approach to the global attractor conjecture, SIAM Journal on Applied Dynamical Systems, 13 (2014), pp. 758-797. 
[25] A. Gorban and G. Yablonsky, Extended detailed balance for systems with irreversible reactions, Chemical Engineering Science, 66 (2011), pp. 5388-5399.

[26] F. Horn And R. Jackson, General mass action kinetics, Archive for rational mechanics and analysis, 47 (1972), pp. 81-116.

[27] B. Joshi AND A. Shiu, Simplifying the jacobian criterion for precluding multistationarity in chemical reaction networks, SIAM Journal on Applied Mathematics, 72 (2012), pp. 857876.

[28] M. Ke, Z. FANG, AND C. GaO, Complex balancing reconstructed to the asymptotic stability of mass-action chemical reaction networks with conservation laws, SIAM Journal on Applied Mathematics, 79 (2019), pp. 55-74.

[29] M. Ke, S. Wu, AND C. GAO, Realizations of quasi-polynomial systems and application for stability analysis, Journal of Mathematical Chemistry, 55 (2017), pp. 1597-1621.

[30] D. Kondepudi And I. Prigogine, Modern thermodynamics: from heat engines to dissipative structures, John Wiley \& Sons, 2014.

[31] K. J. Laidler And M. C. King, Development of transition-state theory, The Journal of physical chemistry, 87 (1983), pp. 2657-2664.

[32] J. C. MAXwell, Illustrations of the dynamical theory of gases. part $i$. on the motions and collisions of perfectly elastic spheres, The London, Edinburgh, and Dublin Philosophical Magazine and Journal of Science, 19 (1860), pp. 19-32.

[33] J. C. MAXwelL, Illustrations of the dynamical theory of gases. part ii. on the process of diffusion of two or more kinds of moving particles among one another, The London, Edinburgh, and Dublin Philosophical Magazine and Journal of Science, 20 (1860), pp. 21-37.

[34] S. RaO, A. VAn der Schaft, and B. Jayawardhana, A graph-theoretical approach for the analysis and model reduction of complex-balanced chemical reaction networks, Journal of Mathematical Chemistry, 51 (2013), pp. 2401-2422.

[35] J. Sethna, Statistical mechanics: entropy, order parameters, and complexity, vol. 14, Oxford University Press, 2006.

[36] G. Shinar, U. Alon, And M. Feinberg, Sensitivity and robustness in chemical reaction networks, SIAM Journal on Applied Mathematics, 69 (2009), pp. 977-998.

[37] G. Shinar and M. Feinberg, Structural sources of robustness in biochemical reaction networks, Science, 327 (2010), pp. 1389-1391.

[38] J. Stoer and C. Witzgall, Convexity and optimization in finite dimensions, (1970).

[39] G. Szederkényi and K. M. Hangos, Finding complex balanced and detailed balanced realizations of chemical reaction networks, Journal of Mathematical Chemistry, 49 (2011), pp. 1163-1179.

[40] D. G. Truhlar, W. L. Hase, and J. T. Hynes, Current status of transition-state theory, The Journal of Physical Chemistry, 87 (1983), pp. 2664-2682.

[41] A. VAn der Schaft, S. RaO, and B. Jayawardhana, On the mathematical structure of balanced chemical reaction networks governed by mass action kinetics, SIAM Journal on Applied Mathematics, 73 (2013), pp. 953-973.

[42] A. VAN DER Schaft, S. RAO, AND B. JaYAwARDhANA, On the network thermodynamics of mass action chemical reaction networks, IFAC Proceedings Volumes, 46 (2013), pp. 24-29.

[43] A. van der Schaft, S. RaO, and B. Jayawardhana, Complex and detailed balancing of chemical reaction networks revisited, Journal of Mathematical Chemistry, 53 (2015), pp. 14451458.

[44] A. Van der Schaft, S. RaO, and B. Jayawardhana, A network dynamics approach to chemical reaction networks, International Journal of Control, 89 (2016), pp. 731-745.

[45] L. WANG, B. MASChKe, AND A. VAN DER SCHAFT, Irreversible port-hamiltonian approach to modeling and analyzing of non-isothermal chemical reaction networks, IFACPapersOnLine, 49 (2016), pp. 134-139.

[46] L. Wang, B. Maschke, And A. VAn Der Schaft, Port-hamiltonian modeling of non-isothermal chemical reaction networks, Journal of Mathematical Chemistry, 56 (2018), pp. 1707-1727.

[47] R. WEGSCHEIDER, About simultaneous equilibria and the relationships between thermodynamics and reaction kinetics of homogeneous systems, Journal of Physical Chemistry, 39 (1902), pp. 257-303.

[48] B. E. Ydstie, Passivity based control via the second law, Computers \& chemical engineering, 26 (2002), pp. 1037-1048.

[49] B. E. Ydstie And A. A. Alonso, Process systems and passivity via the clausius-planck inequality, Systems \& Control Letters, 30 (1997), pp. 253-264. 